Fayetteville State University DigitalCommons@Fayetteville State University

Natural Sciences Faculty Working Papers

College of Arts and Sciences

4-25-1997

\title{
Adiabatic phase transformations in confinement
}

Alexander Umantsev

Fayetteville State University, aumantsev@uncfsu.edu

\section{Recommended Citation}

Umantsev, Alexander, "Adiabatic phase transformations in confinement" (1997). Natural Sciences Faculty Working Papers. Paper 7. http://digitalcommons.uncfsu.edu/natsci_wp/7

This Article is brought to you for free and open access by the College of Arts and Sciences at DigitalCommons@Fayetteville State University. It has been accepted for inclusion in Natural Sciences Faculty Working Papers by an authorized administrator of DigitalCommons@Fayetteville State University. For more information, please contact mlawson@uncfsu.edu. 


\title{
Adiabatic phase transformations in confinement
}

\author{
Alexander Umantsev \\ Department of Materials Science and Engineering, Northwestern University, Evanston, Illinois 60208
}

(Received 11 October 1996; accepted 25 April 1997)

\begin{abstract}
The phase diagram of small one-component particles has been analyzed under conditions of thermal insulation, i.e., conservation of energy. In large isolated systems the absolute stability belongs to heterogeneous states with phase separation. However, for small particles the global stability analysis shows a considerable extension of the single-phase regions into a two-phase zone of the phase diagram. Moreover, for very fine particles with sizes only 5-20 times exceeding interfacial thickness, phase separation does not occur at all and the equilibrium is achieved on homogeneous transition states that can never be obtained in bulk samples because of their absolute instability. The thermodynamic and dynamical explanations are presented. This type of a small-particle phase diagram may be relevant to the theory of amorphization, magnetocaloric effect, and nanophase composite materials where small particles or thin whiskers, capable of undergoing a transition, are immersed into a poorly conducting matrix. In case of small particles of solid solution, where mass conservation replaces the conservation of energy, present results predict the appearance of new stable phases with compositions deeply inside the miscibility gap. (C) 1997 American Institute of Physics. [S0021-9606(97)51129-9]
\end{abstract}

\section{INTRODUCTION}

It has been known for many years that it is possible to produce small metal particles that have structures unparallel to the ones encountered in the bulk. Ultrasmall geometries can force a system to act in ways significantly different to how it behaves without geometrical constraints. Some recent observations even suggest that small particles fluctuate between different (maybe heterogeneous) states, rather than "sit in a fixed position." The unique character of small systems not only provides ways to make new materials but also allows further investigation of the secrets of phase transitions. Until now equilibrium and dynamical properties of systems in confined geometries have been studied mainly in isothermal conditions. However, real transformations rarely occur under conditions of constant temperature because the mechanism of temperature equilibration, that is thermal conductivity, cannot be infinitely fast. It is the intention of the author to analyze the behavior of such systems under the conditions of thermal insulation. This may be the case for composite materials where nanoscale particles or whiskers are immersed in a poorly conducting matrix which makes them effectively insulated, in porous media, or for submicron metal droplets that have been sprayed in high vacuum by some kind of atomization technique. Before dwelling on confining geometries it is advantageous to analyze the basic ideas of transformations in large thermally insulated systems which will be conducive to understanding the peculiarities in small particles.

The basic idea of equilibrium in insulated systems has been formulated by Clausius "in the following simple form: 1. The energy of the universe is a constant. 2. The entropy of the universe tends toward a maximum."1 Later Gibbs developed this idea into an elaborate theory of heterogeneous substances. ${ }^{2}$ However, some points are missing in his theory because Gibbs and followers, e.g., ${ }^{3-7}$ were mainly concerned with the chemical equilibrium of different species as opposed to thermal equilibrium of a one-component system. Whatever the reason is, thus far very little can be found in the literature related to transformations in closed systems. ${ }^{8-11}$ It is the intention of the author partially to cover this gap.

Consider a sample of a high-temperature phase (e.g., water) being cooled down below the melting temperature. So prepared phase is said to be supercooled. A slight fluctuation may initiate a precipitous transition into a low-temperature phase (e.g., ice). However, the course and outcome of such a transition will be different depending upon the type of interactions of the system with the environment. In the system that has been isolated from ambient objects after preparation, the transition is driven by the possibility to increase the total entropy which emerges if energy of the system after preparation is below the equilibrium energy of the hightemperature phase. This energy difference may also be expressed by the deviation of the initial temperature from its equilibrium value, that is the supercooling.

In the next section large isolated systems are considered where the finite size effects are negligible. Although many of these results may be found in Refs. 3-7, we find it appropriate to lay them out here in a form pertinent to the present discussion. Recently ${ }^{12}$ the author analyzed the thermodynamic stability of equilibrium states under conditions of thermal insulation at constant pressure and showed that in the thermodynamic limit, besides homogeneous phases, phaseseparating interfaces may also be locally stable (metastable), i.e., with respect to small perturbations. However, a local analysis is hardly enough, particularly in systems where several different states may be stable under the same conditions. In order to construct the phase diagram one needs to complete the local stability analysis with the global stability analysis, i.e., with respect to all possible variations. In Sec. II the global analysis of stability of states in an isolated system at constant volume is represented in the form of the general 
condition of bifurcation of locally stable states.

A special remark should be made regarding the term isolated system which will be used extensively in our subsequent discussion. According to the first law of thermodynamics the change in the internal energy of a body is a consequence of both heat given to the body and work done on it. In the present study, isolation means that energy of the system is conserved, hence, this implies invariable volume of a system (no external fields) and thermal insulation from the surrounding bodies (no heat fluxes). ${ }^{1,2}$ To specify the interactions of the system with environment, the constraint of constant volume is chosen which makes the present study relevant to transformations in condensed matter (solids and liquids) as opposed to gaseous media where the constraint of constant pressure is more applicable. In fact, of a particular interest for us will be an incompressible motionless medium where work vanishes even for each element of the system. However, internal heat fluxes are permissible during the course of transformation which makes it non-equilibrium and non-adiabatic. A completely adiabatic transformation can be realized in the special material with vanishing thermal conductivity inside (ideal insulator). A transformation will also be adiabatic if it is carried out so fast that different parts of the system have no time for thermal equilibration. ${ }^{13,14}$ Yet, the state of the system after completion of transformation (thermodynamic equilibrium) will be of our concern in the present study. Another remark should be made with regard to the denotations used in the paper: when subscripts and superscripts are used together with thermodynamic functions, the former will refer to the state of the system and the latter to its temperature.

Much progress has been recently made in studying size effects in open systems. These effects can be separated into two different groups: those which are due to the presence of the surface restraining the body, ${ }^{15-18}$ and those which are due to the existence of precursors to the transition in the form of heterophase fluctuations. ${ }^{19,20}$ Usually the former effects can be assessed by the introduction of the "extrapolation length" and vanish at a free surface where the extrapolation length diverges. The latter effects are manifested in the emerging of a narrow region of phase coexistence around the equilibrium temperature and may be addressed by introducing a "scaling ansatz" which specifies the singular part of thermodynamic functions. In the case of the first-order transformations both types of effects yield an equilibriumtemperature shift inversely proportional to the system's size. The present study concentrates on first-order phase transformations in closed systems where phase coexistence is rather a norm than a fluctuation as in open ones. Therefore, of particular interest is the influence of an internal interface separating two coexisting phases. For the outer boundaries we shall assume the free surface conditions. In Sec. III the influence of the interfacial energy of a region that separates the phases on the phase diagram is considered in the capillary approximation which sheds light on the problem of reconstruction of heterogeneous systems in insulation and confinement. Where relevant, juxtaposition with isothermal systems is made.
From the statistical mechanics stand point small isolated systems correspond to a microcanonical ensemble and can be studied by different methods: cluster variation, molecular dynamics, or Monte Carlo for small clusters. In the present paper the mean-field (continuum) method is employed. This method was originally introduced for the analysis of bulk systems undergoing phase transformations but can be successfully extended to small confining thermodynamic systems. On the atomic level, phase transition is viewed as a cooperative motion of atoms which results in the change of atomic configuration in the long range. To characterize this process on the mesoscopic scale, the "coarse-grained" variable is introduced. It is called a transition parameter and designates a degree of transition in a small piece of a material. The transition itself is manifested by the "crossing of a barrier' separating different phases. Same atomic interactions that are responsible for transition make the medium effectively non-local in the sense that there is a certain penalty if neighboring pieces attain different degrees of transformation. Section IV deals with systems of small sizes where creation of an interface is not favorable. As a consequence, a new phase that is prohibited in large systems is found possible. This result is the main thrust of the present paper. In Sec. V comparison with other studies is made which is followed by the discussion in Sec. VI.

\section{ISOLATED SYSTEMS IN THE THERMODYNAMIC LIMIT}

We consider a physical system composed of a large number $N$ of identical particles (typically of the order of $10^{23}$ ) confined to a space of volume $V$. At first the analysis will be carried out in the so-called thermodynamic limit, viz., $N \rightarrow \infty, V \rightarrow \infty$, such that the ratio $N / V$ stays fixed. In this limit, a system possesses two types of properties: extensive, those which are directly proportional to the size of a system, i.e., $N$ or $V$, and intensive, those which are independent thereof. At this point it is advantageous to give a brief definition of a phase. We shall call a phase a homogeneous part of a system distinguishable by a set of intrinsic parameters, which has attained a thermodynamic stability under specified external conditions. The latter may well affect the equilibrium, making different states of the system more favorable. In the present study closed systems are analyzed which are isolated from all external influences. The achievement of local thermodynamic stability does not exclude a possibility for a system to be in a metastable state (metastable phase). Except for homogeneous states, that is phases, various heterogeneous states may be possible. To obtain a complete picture of equilibrium states in an isolated system the analysis of local stability, when a state is being verified with respect to the infinitesimal variations only, should be supplemented with the global stability analysis, when the variations of higher order are not to be neglected. Thus the Gibbsian "Criterion of Equilibrium and Stability" may be reformulated as follows: 


\section{Among all possible states of an isolated system}

the one which has more entropy for the same

energy is the most stable.

This is a criterion of the global stability when the variations of all orders must be taken into account. It may be called the equilibrium selection principle because it allows one to distinguish between several otherwise equivalently stable equilibrium states.

To begin with, we shall analyze the stability of a homogeneous system occupied by one of the possible phases, the number of which, for simplicity, will be reduced to the minimum ( $\alpha$ and $\beta$ ) in this study. Each phase is characterized by its own entropy-versus-energy function $s_{j}\left(e_{j}\right)$, that is Mollier chart, ${ }^{5-7}$ where $s_{j}$ and $e_{j}$ are entropies and energies per unit volume of the phase $j=\alpha$ or $\beta$. We will define the equaentropic point to be the energy density $e_{\alpha \beta}$ such that

$$
s_{\alpha}\left(e_{\alpha \beta}\right)=s_{\beta}\left(e_{\alpha \beta}\right) \text {. }
$$

Without loss of generality, we suppose that $\alpha$-phase is more stable for high energies: $s_{\alpha}>s_{\beta}$ for $e>e_{\alpha \beta}$ and $\beta$-phase is more stable for low energies: $s_{\alpha}<s_{\beta}$ for $e<e_{\alpha \beta}$. Hence, the entropy-versus-energy function of a homogeneous system $s_{H}(e)$ consists of the pieces of the individual curves of $\alpha$ and $\beta$ phases joined at the equaentropic point $e_{\alpha \beta}$, thus having a cusp at the latter (Fig. 1).

Different parts of the system may be occupied by different phases of the matter. If homogeneous phases are com-

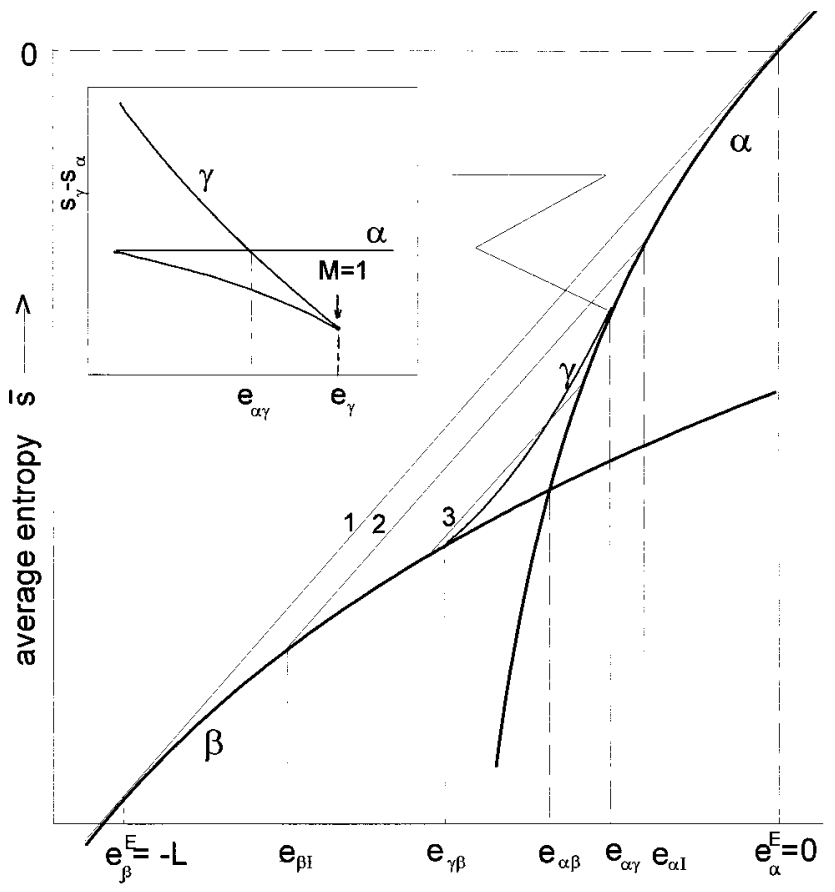

FIG. 1. Mollier chart. Average entropy $\bar{s}$ of a layer as a function of its average energy for different phase states of the thermodynamic system with $Q=0.1$. $\alpha$ and $\beta$-bulk phases; $\gamma$-adiabatic nanophase; $1,2,3$-two-phase states; 1 -very thick layer (thermodynamic limit); 2 -thin layer (capillary approximation); 3-very thin layer (continuum theory). Insert: Difference of the entropy densities of $\gamma$ and $\alpha$ states. The transition state is locally stable beyond the point $M=1$ and globally stable beyond $e_{\alpha \gamma}$. bined, energies of individual phases are added. The entropy of the combined (heterogeneous) system cannot be less than the sum of equilibrium entropies of the separate phases, otherwise phases will stay unrelaxed, see criterion (1). For a sufficiently large system we can ignore the contribution of the internal interface separating the coexisting phases, if such are present, and thermal fluctuations in the system (thermodynamic limit). Then, for the heterogeneous state representing a mixture of $\alpha$ and $\beta$ phases, the condition of equilibrium and stability (1) takes the form

$$
S \equiv s_{\alpha}\left(e_{\alpha}\right) V_{\alpha}+s_{\beta}\left(e_{\beta}\right) V_{\beta} \rightarrow \text { maximum, }
$$

subject to the side conditions of conservation of energy

$$
E \equiv e_{\alpha} V_{\alpha}+e_{\beta} V_{\beta}=\mathrm{const}
$$

and volume

$$
V \equiv V_{\alpha}+V_{\beta}=\text { const } .
$$

The constraints (4) and (5) manifest the absence of energy and mass fluxes into or out of the system and reflect the isolation of our system from ambient bodies. Here, $V_{j}$ is a volume of the phase $j$ which must be neither negative nor greater than the whole volume of the system $V$, yet may vanish. Phase transformation is manifested in the variation of one of these volumes at the expense of the other and may be characterized by the fraction of the phase $\beta$ in the heterogeneous state

$$
\varphi \equiv V_{\beta} / V \text {. }
$$

According to the theorem of the conditional extremum, there exist constants $\lambda$ and $\mu$ (Lagrange multipliers) such that the function $S+\lambda E+\mu V$ has an unconditional extremum. Taking into account that $s_{\alpha}$ is a function of $e_{\alpha}$ only and $s_{\beta}$ is a function of $e_{\beta}$ only we arrive at the following conditions: ${ }^{3-7}$

$$
\begin{aligned}
& -\lambda=\frac{d s_{\alpha}}{d e_{\alpha}}=\frac{d s_{\beta}}{d e_{\beta}}, \\
& -\mu=s_{\alpha}+\lambda e_{\alpha}=s_{\beta}+\lambda e_{\beta} .
\end{aligned}
$$

Besides being general conditions of equilibrium between two bodies that are in contact with each other, Eqs. (7) and (8) determine energy densities of coexisting phases if entropy densities of these phases are known as functions of the former. Equation (7) means that at the energies of coexistence, $e_{\alpha}^{E}$ and $e_{\beta}^{E}$, these functions have same slopes of the tangent lines. Equation (8) means that the coordinates of one phase, e.g., $\left(e_{\beta}^{E}, s_{\beta}^{E}\right)$, belong to the extension of the tangent line to the entropy function of another phase, $s_{\alpha}\left(e_{\alpha}\right)$, at the point of coexistence $\left(e^{E}{ }_{\alpha}, s_{\alpha}^{E}\right)$. Thus at the equilibrium, entropy-density-versus-energy-density functions of coexisting phases are connected by the common tangent. Obviously, the equaentropic point lies between the equilibrium energy densities of the two phases

$$
e_{\beta}^{E}<e_{\alpha \beta}<e_{\alpha}^{E} \text {. }
$$

There is another way of looking at Eqs. (7) and (8): They demonstrate which quantities are actually equilibrated be- 
tween phases at equilibrium. The condition (7) is a standard definition of the temperature of bodies at equilibrium $T_{E} \equiv-1 / \lambda$. The condition (8) demonstrates that at equilibrium the free energy densities of $\alpha$ and $\beta$ phases, defined as $f_{j}=e_{j}-T s_{j}$, are equal. Combined together, Eqs. (7) and (8) allow to determine the equilibrium temperature $T_{E}$ of the coexisting phases ${ }^{3-7}$

$$
f_{\alpha}\left(T_{E}\right)=f_{\beta}\left(T_{E}\right) .
$$

Consider a first-order transformation from a onecomponent phase insulated from other substances. Having initial energy density $e^{0}$, the parent phase may become capable of decomposing into a mixture of phases. As the process in question proceeds under conditions of insulation, the total energy of the system stays constant during the transformation and is determined by the initial energy density of the parent phase

$$
E \equiv e^{0} V \text {. }
$$

Thus Eqs. (3)-(6), (10), and (11) yield the expression for the total entropy of the system after transformation

$$
S_{\alpha+\beta}=\frac{1}{T_{E}}\left[E-f_{\alpha}\left(T_{E}\right) V\right]
$$

and the "lever rule" for the fraction $\varphi$ of the phase $\beta$ in the equilibrium heterogeneous $\alpha+\beta$ mixture

$$
\varphi_{E}=\left\{\begin{array}{l}
1, \quad e^{0} \leqslant e_{\beta}^{E} \\
\frac{e_{\alpha}^{E}-e^{0}}{e_{\alpha}^{E}-e_{\beta}^{E}}, \quad e_{\beta}^{E} \leqslant e^{0} \leqslant e_{\alpha}^{E} . \\
0, \quad e^{0} \geqslant e_{\alpha}^{E}
\end{array}\right.
$$

The total entropy of the mixture is a linear function of the total energy of the system [Eq. (12) and line 1 in Fig. 1] because the temperature is uniform throughout the system and equal to $T_{E}$, Eq. (7). As the fraction of a heterostate must be $0<\varphi<1$, Eq. (13) curtails initial energies of the parent phase that result in the creation of a state with phase coexistence: $e_{\beta}^{E}<e^{0}<e_{\alpha}^{E}$.

Temperature $T$ and the specific heat for constant volume $C_{V}$ of a heterostate are defined as follows: ${ }^{3-7}$

$$
T \equiv\left(\frac{d S}{d E}\right)^{-1} ; \quad V C_{V} \equiv \frac{d E}{d T}=-\left(T^{2} \frac{d^{2} S}{d E^{2}}\right)^{-1} .
$$

They have evident interpretation on the Mollier chart as being inversely proportional to the slope of the tangent line (temperature) and curvature of the plot (specific heat). As a consequence of Eq. (12), a heterostate at equilibrium has the same temperature $T_{E}$ for all energies and possesses an infinitely large specific heat, i.e., may accumulate a certain amount of heat without any temperature raise, although the volume of the system is limited. Obviously, this happens because the fraction $\varphi$ of the state changes.

In the thermodynamic limit entropy and energy are extensive variables, that is they grow linearly with the size of the system if other characteristics, like temperature or fraction, do not change. We shall study now analytical properties of the function entropy-per-unit-volume versus energy-perunit-volume, $\bar{s}(e)$, of the system at equilibrium which is depicted on the Mollier chart. Homogeneous states of the system are represented in this function by their densities $\bar{s}(e)=s_{j}(e)$ and heterogeneous states-by their average quantities: $\bar{s}(e)=S(E / V) / V$, where $E / V=e^{0}$ is the energy density of the parent phase prior to transformation, see Eq. (11). According to the criterion (1), for the decomposition of the phase $\alpha$ to go, it should be that $\bar{s}\left(e^{0}\right)>s_{\alpha}\left(e^{0}\right)$. Invoking Eqs. (7), (8), (10), and (12) and the fact that energy of a stable bulk phase is a growing function of its temperature, we arrive at the condition:

$$
\frac{d s_{\alpha}\left(e_{\alpha}^{E}\right)}{d e_{\alpha}}<\frac{s_{\alpha}\left(e_{\alpha}^{E}\right)-s_{\alpha}\left(e^{0}\right)}{e_{\alpha}^{E}-e^{0}}
$$

which manifests the convexity of the thermodynamic function entropy-versus-energy of the $\alpha$ phase at the equilibrium point $s_{\alpha}\left(e_{\alpha}^{E}\right)$. Evidently, the same analysis is applicable to the phase $\beta$ which results in the convexity of the function $s_{\beta}\left(e_{\beta}^{E}\right)$ at the equilibrium point. All pieces of the function $\bar{s}(e)$ that belong to homogeneous phases $\alpha$ and $\beta$ are properly convexed which is guaranteed by the positivity of the specific heat, Eq. (14). However, the function $s_{H}(e)$, which consists of the "homogeneous pieces" of $\alpha$ and $\beta$ phases joined together at the equaentropic point, is not convex in the vicinity of this point. To convexify this function the common tangent construction, Eqs. (7), (8), (12) and (13) are used. The physical meaning of this procedure consists in the separation of the system into a heterogeneous mixture of two phases. Then the condition of equilibrium and stability (3)(5) takes the form

$$
\bar{s}\left[\varphi e_{1}+(1-\varphi) e_{2}\right] \geqslant \varphi \bar{s}\left(e_{1}\right)+(1-\varphi) \bar{s}\left(e_{2}\right) .
$$

This condition means that in the thermodynamic limit $\bar{s}(e)$ is a convex upward function of energy. Equality here is attained at the equilibrium fraction $\varphi_{E}$, Eq. (13). Thus one can see that the average-entropy versus average-energy function $\bar{s}(e)$ is convex upward everywhere.

The basic features of transformations in isolated systems can be elucidated on the Mollier chart (entropy-versusenergy plot, Fig. 1) where entropies of different possible states are represented as functions of their energies on a perunit-volume basis. For definiteness, below will be considered a transformation from a high energy phase $\alpha$. The curves representing homogeneous phases intersect at the equaentropic point $e_{\alpha \beta}$, see Eq. (2). The straight line representing the $\alpha+\beta$ mixture, Eq. (12), is tangential to both single-phase curves. The points of tangency $e_{\alpha}^{E}$ and $e_{\beta}^{E}$ and the equaentropic point $e_{\alpha \beta}$ break the energy axes down into four different regions, cf. Eq. (9): (i) For the average energy density above $e_{\alpha}^{E}$ the transformation does not go and the system stays in the parent phase; (ii) For the average energy density between $e_{\alpha}^{E}$ and $e_{\alpha \beta}$ the system decomposes into two phases $\alpha$ and $\beta$ of fixed energies $e_{\alpha}^{E}$ and $e_{\beta}^{E}$, and their relative proportions are given by the lever rule, Eq. (13); (iii) For the average energy density between $e_{\alpha \beta}$ and $e_{\beta}^{E}$ the equilibrium state 
also contains two phases $\alpha$ and $\beta$ of fixed energies $e_{\alpha}^{E}, e_{\beta}^{E}$ and relative proportion $\varphi_{E}$. However, the transformation path may pass through the metastable homogeneous state $\beta$ with the temperature above equilibriumal, and the system may be trapped there. The real scenario of transformation depends upon kinetic properties of the system. (iv) For the average energy density below $e_{\beta}^{E}$, only phase $\beta$ will be present in the system after transformation. It is curious to inquire why in the regions (i) and (iv) only "pure," that is unmixed, states exist, although the straight line corresponding to the $\alpha+\beta$ mixture lies above the individual curves of pure states. The answer is in the lever rule (13), which gives negative fraction $\varphi$ for the region (i) and $\varphi>1$ for the region (iv).

It must be emphasized here that the described geometrical analysis of an adiabatic system is completely analogous to the common tangent construction in the thermodynamics of binary alloys where the Gibbs free energy of alloys substitutes entropy of an adiabatic system and mass conservation of one of the components substitutes the energy conservation. Transformation in the region (iii) is reminiscent of the solute trapping and in the region (iv) of the massive transformation in alloys.

\section{A. General criterion of bifurcation}

According to the above formulated criterion of the global thermodynamic stability (1), a new state will "branch off' of the homogeneous parent phase $\alpha$ when the entropy of the former becomes greater than that of the latter for the same energy of the system. On the Mollier chart a phase transition is manifested by the bifurcation of the line of a new state from the line of the parent one. As the size of the system becomes smaller its phase diagram becomes more complicated because several different homogeneous and heterogeneous states may branch off the parent phase at different energies. There are many different ways how one can characterize the energy of a system under consideration. It is generally accepted that the best way to deal with a onecomponent thermodynamic system is to set its energy and entropy as functions of temperature and specific volume, if the latter varies. Below we shall describe the outcome of transformations in insulated systems of different sizes in terms of the initial $T^{0}$ and final $T^{*}$ temperatures where $T^{0}$ is the temperature of a homogeneous parent phase $\alpha$ prior to transformation. To find the bifurcation parameters, temperatures of the initial $T^{0}$ and final $T^{*}$ states, we shall introduce the excess (singular) quantities of energy, entropy, freeenergy as the differences between the actual thermodynamic functions of the system at equilibrium and those that the homogeneous parent phase would have if it occupied the entire volume of the system with the temperature equal to the actual one and uniform throughout ${ }^{2}$

$$
\Delta\left\{\begin{array}{c}
E \\
S \\
F
\end{array}\right\}=\left\{\begin{array}{l}
E \\
S \\
F
\end{array}\right\}_{E}(T, V)-V \times\left\{\begin{array}{l}
e \\
s \\
f
\end{array}\right\}_{\alpha}(T) .
$$

Such definitions are inspired by the fact that temperatures of all the components of a heterostate at equilibrium are equal, Eq. (7). For homogeneous states we may use the excess densities $\Delta\{e, s, f\}$, that is, the excess quantities per unit volume.

$\Delta F(T, V)$ may be either positive or negative. A transformation into a state with $\Delta F<0$, as exemplified by the homogeneous $\alpha \rightarrow \beta$ transformation at temperatures below $T_{E}$, may occur in an open, that is, isothermal, system. In this case, $-\Delta F / V$ is called the driving force and is usually proportional to $\left(T_{E}-T\right)$. On the other hand, a state with $\Delta F(T, V)>0$ must not be a terminal state of transformation in an open system of temperature $T$ and may serve only as an activation barrier for such reaction. However, in an isolated system a state with $\Delta F>0$ may be the global optimizer, i.e., the end point of a transformation.

To elucidate the latter assertion, we shall formulate the general criterion of bifurcation of a new state (either homogeneous or heterogeneous) from the parent phase $\alpha$. To begin with, notice that as the total energy is conserved

$$
e_{\alpha}(T)=e^{0}-\frac{\Delta E(T, V)}{V},
$$

where $T$ is the transformed-state temperature. Then, expanding the function $s_{\alpha}(e)$ into the Taylor series and using the definitions of temperature and specific heat (14) we obtain:

$$
\begin{aligned}
s_{\alpha}(e)= & s_{\alpha}\left(e^{0}\right)-\frac{\Delta E}{V T^{0}}-\frac{1}{2 C_{V \alpha}}\left(\frac{\Delta E}{V T^{0}}\right)^{2} \\
& +O\left[\left(\frac{\Delta E}{C_{V \alpha} V T^{0}}\right)^{3}\right] .
\end{aligned}
$$

On the premise of a small excess internal energy of the transformation $\Delta E$ as compared to the thermal energy of the parent phase $C_{V \alpha} T^{0} V$, substitution of this relation into the definition of the excess quantities (16) yields the expression:

$$
\begin{aligned}
\bar{s}\left(e^{0}, V\right)-s_{\alpha}\left(e^{0}\right)= & -\frac{\Delta F}{V T^{*}}+\frac{1}{2 C_{V \alpha}}\left(\frac{\Delta E}{V T^{*}}\right)^{2} \\
& +O\left[\left(\frac{\Delta E}{C_{V \alpha} V T_{E}}\right)^{3}\right] .
\end{aligned}
$$

Here, $s_{\alpha}\left(e^{0}\right)$ is the entropy density of the initial state after the preparation but before the transformation. For a transformation into a new state to go, $\bar{s}\left(e^{0}, V\right)$ must be greater than $s_{\alpha}\left(e^{0}\right)$. This is true for any state with $\Delta F<0$ even if $\Delta E=0$. An example of such transformation will be encountered in the next section. However, Eq. (18) shows that there may exist an adiabatic transformation into a state with $\Delta F>0$ which will occur only if the negative contribution to the entropy due to the free-energy excess is offset by the positive contribution due to the internal energy excess. At the bifurcation point $\left(\bar{s}-s_{\alpha}^{0}\right)$ vanishes. Hence, transformation into a state with $\Delta F>0$ requires the threshold energy $\Delta E$ which may be found from the following equation:

$$
\Delta E_{\mathrm{th}}^{2}\left(T^{*}\right)=2 C_{V \alpha} V T^{*} \Delta F\left(T^{*}\right) .
$$


This equation may also be used to determine the bifurcation temperature $T^{*}$ of the transformed state with $\Delta F>0$ while temperature or energy of the parent phase at the start of the transformation is determined by Eq. (17). Notice that the threshold energy of such bifurcation obeys the constraint

$$
0<\Delta F \ll\left|\Delta E_{\text {th }}\right| \ll C_{V \alpha} T^{0} V .
$$

If more than one state branches off the parent phase then, to assess the regions of their global stabilities, one needs to apply the criterion (1) to these states directly because the criterion (17) and (19) is not applicable. However, there is a way one can determine the global stabilities of two states, different from $\alpha$, and make use of the criterion (17) and (19). Indeed, the state that branches off the $\alpha$ phase at larger energy is more stable around this bifurcation point than another state that branches off at smaller energy. Apparently, at still smaller energies there might appear to be another bifurcation and these states may exchange their stabilities. This wraps up the formulation of the general criterion of the global stability of states as represented by the simple one-component isolated system. Below this criterion will be applied to different situations.

\section{B. “Linear' thermodynamic system}

It may be of interest to look at an example of a thermodynamic system with equal and temperature-independent specific heats in both phases $C_{V \alpha}=C_{V \beta}=C$. Such a system can be called linear. For the temperature of the parent phase at the bifurcation in the linear system, Eqs. (14), (17) and (19) yield

$$
T^{0}=T^{*} \pm \sqrt{\frac{2 T^{*} \Delta F\left(T^{*}\right)}{C V}} .
$$

Note that at the bifurcation, temperature of the system changes discontinuously by the jump from $T^{0}$ to $T^{*}$.

As the entire volume of the system $V$ remains unchanged, the latent heat at an arbitrary temperature can be determined as follows: $3,4,13$

$$
L(T) \equiv e_{\alpha}(T)-e_{\beta}(T) .
$$

Differentiating Eq. (22) with respect to $T$ and keeping the volume constant, we obtain the formula for the temperature dependence of the latent heat

$$
\frac{d L}{d T}=C_{V \alpha}(T)-C_{V \beta}(T)
$$

which shows that the latent heat of the linear system is a constant. This relation is analogous to the Planck's formula for open systems. ${ }^{3,4}$ Notice that in general the latent heat cannot be determined via entropy differences of phases because $s_{\alpha}(T)-s_{\beta}(T) \neq L(T) / T$ at arbitrary temperature. The latent heat may be either positive: exothermic reaction, or negative: endothermic reaction. Although both cases are possible, in the present study we are concerned mostly with the former one which entails negative values of $\Delta E$ for any heterostate where $\alpha$ and $\beta$ phases are mixed together in an equilibrium proportion. Therefore, for exothermic transformation one should choose a negative sign in Eq. (21).

It is advantageous to choose as the standard state of the linear system the parent phase $\alpha$ at the equilibrium temperature $T_{E}:\left(e_{\alpha}^{E}=0, s_{\alpha}^{E}=0\right)$ and reckon energy and entropy from this state. Then

$$
\begin{aligned}
& e_{\alpha}(T)=C\left(T-T_{E}\right) ; \quad s_{\alpha}(T)=C \ln \frac{T}{T_{E}} ; \\
& s_{\alpha}(e)=C \ln \left(1+\frac{e}{C T_{E}}\right) .
\end{aligned}
$$

Notice that, as a result of such choice of the standard state, the free energy of the parent state and its first derivative with respect to temperature vanish at the equilibrium point. The approximation of the temperature independent specific heat simplifies our calculations in the vicinity of $T_{E}$ but, apparently, cannot be extended to very low temperatures. For the product phase this yields

$$
\begin{aligned}
& e_{\beta}(T)=C\left(T-T_{E}\right)-L ; \quad s_{\beta}(T)=C \ln \frac{T}{T_{E}}-\frac{L}{T_{E}}, \\
& s_{\beta}(e)=C \ln \left(1+Q+\frac{e}{C T_{E}}\right)-\frac{L}{T_{E}} ; \quad Q=\frac{L}{C T_{E}} .
\end{aligned}
$$

Parameter $Q$, which is the ratio of the latent heat $L$ to the thermal energy density $C T_{E}$ of the standard state, classifies different transformations as weak, small $Q$, or strong, large $Q$.

Energy of the equaentropic point and $T_{\alpha \beta}^{0}, T_{\alpha \beta}^{*}$ of a homogeneous bifurcation from $\alpha$ to $\beta$ phase can be found by the direct application of Eq. (2) to the linear system (23) and (24). This yields

$$
\begin{aligned}
& e_{\alpha \beta}=-C T_{E}\left(1-\frac{Q}{\exp Q-1}\right) ; \\
& T_{\alpha \beta}^{0}=T_{E} \frac{Q}{\exp Q-1}<T_{E} ; \quad T_{\alpha \beta}^{*}=T_{E} \frac{Q \exp Q}{\exp Q-1}>T_{E} .
\end{aligned}
$$

In the case of a weak transformation, which will be of special interest for the discussion below, $e_{\alpha \beta} \approx-L / 2, T_{\alpha \beta}^{0} \approx T_{E}$ $-L / 2 C, T^{*}{ }_{\alpha \beta} \approx T_{E}+L / 2 C$.

The average energy of the linear system may be conveniently expressed through the dimensionless supercooling $\Delta \theta$ as follows:

$$
\Delta \theta \equiv\left(e_{\alpha}^{E}-e_{\alpha}^{0}\right) / L=C\left(T_{E}-T^{0}\right) / L .
$$

In alloys the role of supercooling is played by the supersaturation normalized by the width of the miscibility gap. The temperature of the final state $T^{*}$ after transformation is greater than that of the initial one $T^{0}$. There may be a special reason why this temperature may attain the equilibrium value: $T^{*}=T_{E}$. Then the bifurcation criterion (17) and (19) is greatly simplified and may be expressed in terms of the threshold supercooling

$$
\Delta \theta_{\mathrm{th}}=\sqrt{\frac{2 \Delta F\left(T_{E}\right)}{Q L V}} .
$$


Application of these ideas to the transformation in the thermodynamic limit yields a trivial result that $T^{0}=T^{*}=T_{E}$, and $\Delta \theta_{\mathrm{th}}=0$ because $\Delta F=0$. Depending upon the initial temperature $T^{0}$, the $\alpha$ phase may either stay untransformed (globally stable phase), decompose into a mixture of coexisting phases $\alpha$ and $\beta$, or transfer completely into another, more stable, phase $\beta$. Decomposition starts "immediately" after the supercooling of the parent phase $(\Delta \theta>0)$ and goes until the magnitude of supercooling attains unit $(\Delta \theta=1)$. For the equilibrium transformation fraction of the heterostate Eqs. (13), (22), and (26) yield

$$
\varphi_{E}=\Delta \theta \text {. }
$$

For supercoolings higher than one parent phase will transform entirely into the product phase $\beta$.

\section{INFLUENCE OF INTERNAL INTERFACE: THE CAPILLARY APPROXIMATION}

In the previous section we have analyzed the equilibrium states of an isolated system in the thermodynamic limit of infinitely large sizes where influence of a phase-separating interface is negligible. Now we intend to assess the size effect in isolated systems. We envisage an internal interface as a thin layer of the area $A$ and thickness $l_{I} \ll V / A$ that separates two coexisting phases, encompasses rapid variations of different physical properties between phases and is no obstacle to the energy fluxes. For a more elaborate description of an interface one may inquire in Refs. 2 and 4.

The entropy $s_{I}$ attributed to the unit area of the interface is a function of the internal energy $e_{I}$ of the same piece of interface and is independent of the energies of phases $\alpha$ and $\beta$ that it separates. However, both $s_{I}$ and $e_{I}$ may depend upon the total volume of the system $V$. The interfacial area will be deemed dependent on the volume of the product phase $V_{\beta}$ only. All this changes the total entropy $S$ of the system by the amount $s_{I}\left(e_{I}, V\right) A$ and the total energy $E$ by the amount $e_{I}(V) A$, cf. Eqs. (3) and (4), but does not change the total volume because we assume that $V_{I}=0 \quad\left(N_{I}=0\right)$. Then application of the method of Lagrange multipliers to the condition of equilibrium and stability (3)-(5) yields

$$
\begin{aligned}
-\lambda & =\frac{d s_{\alpha}}{d e_{\alpha}}=\frac{d s_{\beta}}{d e_{\beta}}=\frac{d s_{I}}{d e_{I}}, \\
-\mu & -\frac{\partial\left(s_{I}+\lambda e_{I}\right)}{\partial V} A \\
& =s_{\alpha}+\lambda e_{\alpha}=s_{\beta}+\lambda e_{\beta}+\left(s_{I}+\lambda e_{I}\right) \frac{d A}{d V_{\beta}} .
\end{aligned}
$$

Equation (29) shows that the introduction of an interface does not change equality of temperatures of the phases $\alpha$ and $\beta$ and allows to introduce the temperature of an interface $T_{I} \equiv\left(d s_{I} / d e_{I}\right)^{-1}$, so that temperatures of all parts of the system are equilibrated: $T_{I}=T_{\alpha}=T_{\beta}$. Equation (30) shows that the size dependence of interfacial entropy and energy alone do not change energies of coexistence $e_{\alpha}^{E}, e_{\beta}^{E}$ and the equilibrium temperature $T_{E}$. However, the dependence of $A$ on
$V_{\beta}$ alters the equilibrium. Using the definition of the free energy, the equilibrium conditions may be expressed as follows:

$$
f_{\alpha}(T)=f_{\beta}(T)+\sigma(T, V) \frac{d A}{d V_{\beta}} ; \quad \sigma \equiv f_{I}(T, V)=e_{I}-T s_{I} .
$$

Here, $\sigma$ is the free energy of the unit area of the interface, the surface tension, which is assumed to be positive. The area of a regular (nonfractal) surface obeys a power law as a function of the encompassed volume: $A=K_{D} V_{\beta}^{1-1 / D}$, where $D$ is the dimensionality of the space. Then, substitution of Eq. (31) into Eq. (16) yields for the excess free energy of equilibrium heterogeneous mixture of $\alpha$ and $\beta$ phases: $\Delta F=\sigma A / D>0$. Application of the general criterion of bifurcation (17) and (19) shows that adiabatic transformation into such a state always requires the threshold energy per unit volume

$$
\frac{\left|\Delta E_{\mathrm{th}}\right|}{V}=\sqrt{2 C T^{*} \sigma \frac{A}{D V}}
$$

In what follows only a one-dimensional (1D) case will be considered where the phase-separating interface is a plane. So, we postulate that the heterogeneous layer is completely confined between the geometrical surfaces $x=0$ and $x=X$ parallel to the interface, where $V=X A$. Great simplification of this problem stems from the independence of $A$ from $V_{\beta}$ which results in the equality of the free energy densities of $\alpha$ and $\beta$ phases at equilibrium, Eq. (31), hence, the temperature of the heterostate is $T_{E}$, Eq. (10). Then conditions (29) and (30) allow us to express the entropy of a two-phase layer reckoned from the $\alpha$ phase at $T_{E}$ as a function of the energy of the system reckoned from the same state and the equilibrium value of the transformation fraction as a function of the supercooling

$$
S\{\alpha+I+\beta\}=\frac{E-\sigma A}{T_{E}} ; \quad \varphi_{E}\{\alpha+I+\beta\}=\Delta \theta+\frac{e_{I}}{L X} .
$$

These relations show that the interfacial contribution does not break a linear dependence between the average energy $e^{0}=E / X A$ and entropy $\bar{s}=S\{\alpha+I+\beta\} / X A$ of a two-phase system at equilibrium, but introduces a constant shift inversely proportional to the system size $X$ [Fig. 1, line 2; cf. line 1 and Eq. (12)]. Comparing Eq. (33) with Eq. (28) one can see that transformation in a finite-size system yields larger fraction of the low energy phase than that in the thermodynamic limit, unless the surface energy vanishes.

To find the supercooling at which this state bifurcates off the parent phase $\alpha$ we shall apply the general criterion of bifurcation, as expressed by Eqs. (17)-(21), to the finite-size 1D system. In the capillary approximation $\Delta F=\sigma A$. The fact that temperature of the heterostate $\{\alpha+I+\beta\}$ is $T_{E}$ brings a great simplification to our task because Eq. (27) can be used. Thus, for the threshold supercooling and energy at the beginning of transformation Eqs. (17), (19), and (27) yield 


$$
e_{\alpha I}=-L \Delta \theta_{\alpha I} ; \quad \Delta \theta_{\alpha I}=\sqrt{2 \frac{l_{C}}{X}} ; \quad l_{C}=\frac{\sigma C T_{E}}{L^{2}} .
$$

After the transformation, temperature of the $\alpha$ phase returns to the equilibrium value: $T^{*}=T_{E}$ and there appears a new phase $\beta$ at the same temperature. Therefore, the reaction at bifurcation can be expressed as follows: $\alpha\left(T_{\alpha I}^{0}\right) \rightarrow\{\alpha+I+\beta\}\left(T_{E}\right)$, where $T_{\alpha I}^{0}=T_{E}-L \Delta \theta_{\alpha I} / C$.

This result shows that the necessity to create an interface substantially retards the transformation in isolated systems. Several comments should be made with regard to Eqs. (34). First, $\Delta \theta_{\alpha I}$ manifests a genuine supercooling of a parent phase due to the finite dimension of the system rather than a shift of the phase diagram. Second, we have to emphasize that the adiabatic shift scales as $X^{-1 / 2}$ and constitutes a surprisingly strong effect as compared to the isothermal one ${ }^{17-20}$ which scales as $X^{-1}$. This happens because an equilibrium in an adiabatic system involves a phase-separating interface which is not a part of equilibrium under isothermal conditions. The isothermal size effect is completely due to the change of the microscopic interaction at the surface as compared to the bulk. Theoretically this is manifested in the introduction of the "extrapolation length," so that in a sample with a free surface, i.e., noninteracting with the ambience, where extrapolation length diverges, the isothermal finite size effect would vanish completely. On the contrary, in adiabatic systems the size effect is present although the surface prevents any microscopic interactions between the system and environment, e.g., the energy flux is absent, so that the system should draw resources for transformation from itself. This delays a transformation until higher driving forces and causes a supercooling. Third, one may notice that to characterize a finite-size threshold supercooling in an isolated system a new length scale $l_{C}$ comes about which is called the capillary length. For weak transformations the capillary length may be many times larger than the interatomic distance $l_{A}=\sigma / L$. The same length scale, $l_{C}$, determines the Gibbs-Thompson effect or an equilibrium-temperature change due to the curvature of an interface in manydimensional transformations. There are, however, important differences between the former and the latter. The presence of a curved interface entails the free energy change of the minority phase, see Eq. (31), and a shift of the equilibrium temperature, while the flat interface does not alter the equilibrium temperature of the $1 \mathrm{D}$ isolated system, that is $T^{*}=T_{E}$, but the start of transformation is retarded, that is $T_{\alpha I}^{0}<T_{E}$. It is also of interest to note that a spherical nucleus of the minority phase has different internal pressure and, at most, is in unstable equilibrium with the majority matrix in an isothermal system, while a heterogeneous mixture of these phases separated by a flat interface constitutes a global optimizer of an adiabatic system.

Obviously, much the same way as for the start of transformation, one can obtain analogous expressions for the bifurcational energy $e_{\beta I}$, supercooling, and fraction at the end of transformation or merging of the heterostate line with the line of $\beta$-phase

$$
e_{\beta I}=-L-e_{\alpha I} ; \quad \Delta \theta_{\beta I}=1-\Delta \theta_{\alpha I} ; \quad \varphi_{\beta I}=1-\varphi_{\alpha I}
$$

This expression concludes the study of the influence of an internal interface on phase transformations in isolated finite-size systems and demonstrates that energies of the beginning and end of transformation deviate from the equilibrium ones in the thermodynamic limit, thus decreasing the coexistence region. In Fig. 1 is depicted the equilibrium average-entropy-versus-average-energy function $\bar{s}(e, X)$ of the finite-size system. It is represented by the "homogeneous pieces" of the $\alpha$ and $\beta$ curves for the energies above $e_{\alpha I}$ and below $e_{\beta I}$ respectively, and by the "heterogeneous straight line 2 " in between. Notice that in the vicinity of the bifurcational energies $e_{\alpha I}$ and $e_{\beta I}$ convexity of the curve $\bar{s}(e, X)$ is violated. This occurs not because the intrinsic properties of individual phases change but because combining individual phases in a finite-size system may decrease its entropy after relaxation to equilibrium. The latter is due to the creation of a phase-separating interface which, so to speak, arrests more energy than releases entropy, Eqs. (33).

The phase diagram is a network of lines in the controlparameters space of a system that separate domains where homogeneous stable states (phases) exist from the domains where these states coexist. In Fig. 2 the phase diagram of a finite-size system is depicted in the plane (average energy, size) where the bifurcation conditions (34) and (35) are represented by the curve $X^{*}(e)$ that has two branches. The twophase $\{\alpha+I+\beta\}$ region is separated from the single-phase ones by the low-energy branch on the $\beta$-side, Eq. (35), and the high-energy branch on the $\alpha$-side, Eq. (34). Line 2

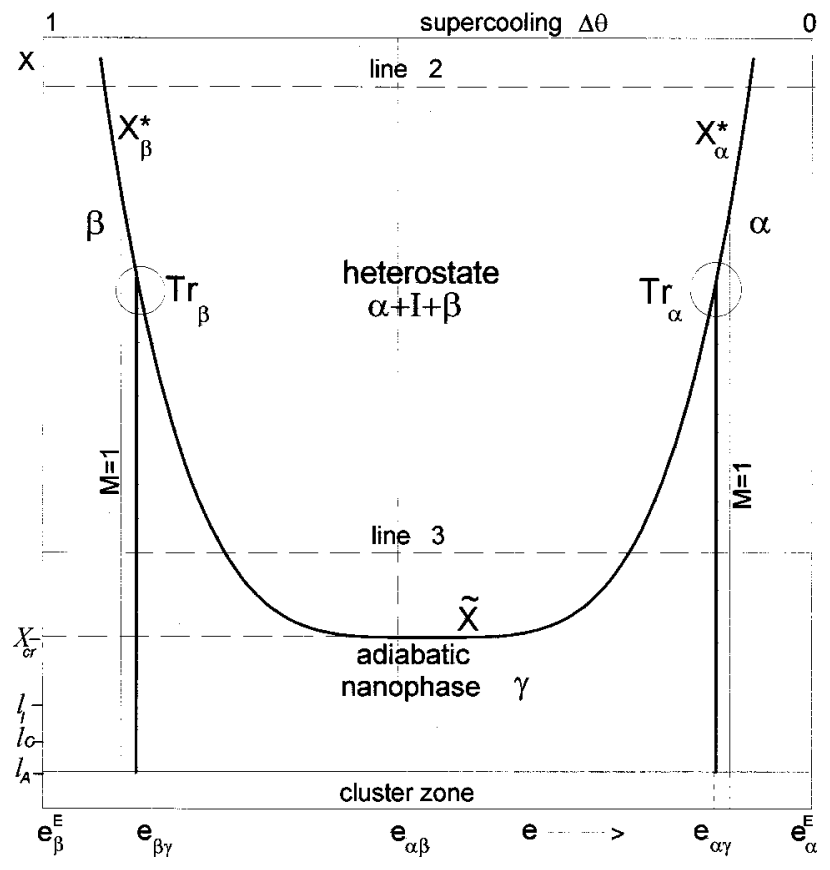

FIG. 2. Phase diagram of a finite-size adiabatic system in the plane energydensity versus size. Hard lines-phase boundaries $X_{\alpha}^{*}(e)$, Eq. (34); $X_{\beta}^{*}(e)$, Eq. (35); $\widetilde{X}(e)$, Eq. (65); $e_{\alpha \gamma}$, Eq. (62); $e_{\gamma \beta}$. 
corresponds to the finite-size system of the linear dimension $X$ and the thermodynamic limit is not shown in Fig. 2.

Equations (34) and (35) also allow another interpretation: temperatures of adiabatic transitions in opposite directions do not coincide in systems of finite size, e.g., melting and freezing temperatures are not equal any more. This manifests a hysteresis in a transformation behavior of such a system when "freezing" starts at temperature $T_{f}<T_{E}$ and "melting" starts at temperature $T_{m}>T_{E}$. For the width of the hysteresis loop Eqs. (34) and (35) yield

$$
\frac{T_{m}-T_{f}}{T_{E}}=\sqrt{\frac{8 \sigma}{C T_{E} X}} .
$$

\section{A. Partial equilibrium in isolated systems}

Equations (29) and (30) constitute conditions of the full equilibrium in the system: The former means that temperatures of the interface and both phases are equilibrated and the latter implies that the temperature takes on a particular value of $T_{E}$. However, in a real transformation these two different processes may have different time scales. Therefore it is of interest to analyze a partially equilibrated system when temperatures of both phases are equal [Eq. (29) is fulfilled] but not to the equilibrium temperature [Eq. (30) or Eq. (8) is not fulfilled]: $T_{\alpha}=T_{\beta}=T_{I} \neq T_{E}$. As the same definitions, Eqs. (14), may be used to determine temperature and specific heat of a heterogeneous state away from equilibrium, the average entropy of a 1D linear system as a function of average energy (supercooling) and transformation fraction may be expressed as follows:

$$
\frac{s_{X}(\Delta \theta, \varphi)}{C}=\ln \left[1+Q\left(\varphi-\Delta \theta-\frac{e_{I}}{L X}\right)\right]-Q \varphi-\frac{s_{I}}{X C} .
$$

In Fig. 3 the quantity $s_{X} / C$ is depicted as a function of the transformation fraction $\varphi$ for an infinitely large system (hard line) and for a layer of finite thickness $X$ (dashed curve). Analysis of the function (37) shows that $s_{\infty}(\Delta \theta, \varphi) / C$ attains a maximum at $\varphi=\Delta \theta$, which coincides with Eq. (28). The shape of the curve $s_{X}(\Delta \theta, \varphi) / C$ is similar to that of the infinite system with the difference in the position of the maximum: It is lower on the amount $s_{I} / C X$ and displaced on the amount $e_{I} / L X$ in the direction of greater fractions, cf. Eqs. (33) and (37). Notice that in the domains $0<\varphi<\varphi_{\alpha I}$ and $\varphi_{\beta I}<\varphi<1$ this function falls below the entropy of the corresponding homogeneous phase, that is $s_{X}$ is not convex as a function of $\varphi$ because the functional space of a finite-size system is non-convex. Simply phrased, one can say that in these domains the decomposition will not go because the system cannot support heterostates with $0<\varphi<\varphi_{\alpha I}$ and $\varphi_{\beta I}<\varphi<1$.

The above described analysis of the finite-size closed systems may fail in case of very thin layers (small particles) because some of the assumptions made are not valid. For instance, if the interfacial thickness $l_{I}$ is comparable with the system size $X$, some space should be allocated for it which will change the transformation fraction $\varphi$. Looking at the expression (34) one can conclude that the assumption of

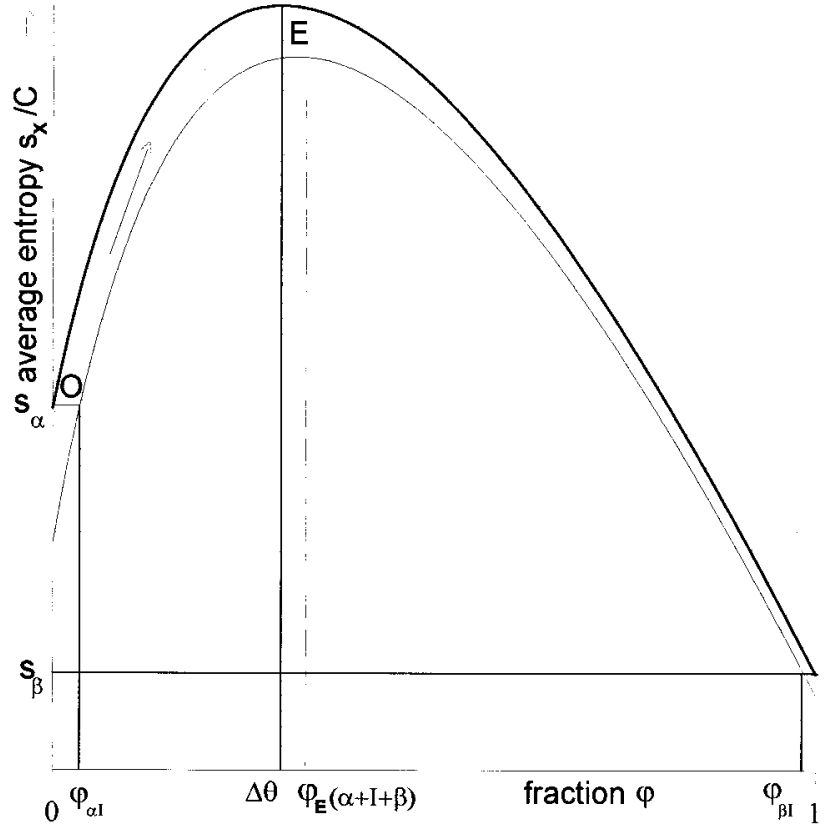

FIG. 3. Average entropy $s_{X}$ of a layer as a function of the fraction $\varphi$ for the initial supercooling $\Delta \theta=0.3$ and different thicknesses of layers $X$ : hard line-very thick layer (thermodynamic limit); solid line-thin layer, $\sigma / L X=0.05$ (capillary approximation).

small bifurcation energy $e_{\alpha I}$ is satisfied in finite but not very thin layers. The interfacial energy may change as a result of interacting with the boundaries. And finally, $\alpha$ and $\beta$ phases themselves may alter in a confinement which will entail change of the temperature of their coexistence.

\section{SMALL PARTICLES: THE CONTINUUM APPROACH}

\section{A. Transition state}

As the system transforms from the initial state to the final state it passes through a continuous series of intermediate configurations characterized by different magnitudes of internal parameters and associated values of thermodynamic functions. It is assumed that one of these intermediate configurations - the transition or surface state-is a quasiequilibrium, labile (according to Ostwald's classification) state $\gamma$ which has unique values of these functions. From the microscopic stand point in the transition state atoms do not occupy stable positions but are in activated configurations, that is "half-way" from one stable configuration $\alpha$ to another $\beta$. The excess of the free-energy density of the transition configuration $\Delta f_{\gamma} \equiv f_{\gamma}-f_{\alpha}$ is known as the activation barrier and should be positive, otherwise the $\alpha \rightarrow \beta$ transformation is barrierless. A transition-state configuration $\gamma^{T}$ can be achieved in adiabatic transformation from the initial state $\alpha^{0}$ only if energies of these states are equal: $e_{\alpha}^{0}=e_{\alpha}^{T}+\Delta e_{\gamma}^{T}$ [see Eq. (17)]. Importantly, there may be conditions in the system when the parent phase becomes metastable with respect to transformation into the homogeneous transition state $\left(\alpha^{0} \rightarrow \gamma^{T}\right)$, no matter how strange this may sound. Indeed, for certain energies of the system, the entropy of the transition state $\gamma^{T}$ exceeds that of the parent phase $\alpha^{0}$ although 
their energies are equal. For this to occur the parent phase even needs not to be unstable and, in fact, may only be slightly supercooled below the equilibrium point. In this case, conceivably, there is another state that separates these two and serves as a barrier for transformation.

The excess free energy density of a system, capable of phase transition, contains two different energy scales: the latent heat $L \equiv \Delta e_{\beta}\left(T_{E}\right)$, Eq. (22), and the activation barrier $B \equiv \Delta f_{\gamma}\left(T_{E}\right)$ at the equilibrium temperature. On the mesoscopic level of description these two scales should be considered independent. In the present study we are concerned with exothermic reactions from a higher symmetry $\alpha$-phase to a lower symmetry $\beta$-phase where $\Delta s_{\beta}^{E}=-L / T_{E}$. Crystallization may be an example of that. This suggests that $\Delta s_{\gamma}^{E} \approx-\frac{1}{2} L / T_{E}$ and $\Delta e_{\gamma}^{E} \approx B-\frac{1}{2} L$ because, according to the definition, the transition state is "half-way" between $\alpha$ and $\beta$. Of particular interest for the present discussion is a weak first-order transition where $L \ll C T_{E}$. For the $\alpha^{0} \rightarrow \gamma^{T}$ transition in such system to occur, inequality (20) must hold: $B \ll\left|\Delta e_{\gamma}^{E}\right| \ll C T_{E}$, that is both energy scales are much smaller than the average thermal energy

$$
B \ll L \ll C T_{E} .
$$

The bifurcation temperatures of such transformation in a linear system may be found from Eq. (17)

$$
T_{\alpha \gamma}^{0}=T_{\alpha \gamma}^{*}+\frac{\Delta e_{\gamma}\left(T_{\alpha \gamma}^{*}\right)}{C} .
$$

The activation configuration in the system, where conditions (38) are fulfilled, is less energetic than the initial one would have been at the same temperature because $\Delta e_{\gamma}^{E}<0$. Hence the temperature of the activated state $T_{\alpha \gamma}^{*}$ is higher than that of the parent phase $T_{\alpha \gamma}^{0}$ and may be near the equilibrium value $T_{E}$. Accepting the approximation $T_{\alpha \gamma}^{*}=T_{E}$, Eq. (19) yields for the bifurcational energy

$$
e_{\alpha \gamma} \approx-\sqrt{2 C T_{E} B} .
$$

At energies less than $e_{\alpha \gamma}$ the transition state is more stable than the $\alpha$-phase but may be less stable than the $\beta$-phase. To assess the stability of the transition state with respect to the $\beta$-phase one needs to apply the criterion (3)-(5) to the latter. However, the above formulated criterion (17)-(20) allows one to determine whether the $\gamma$-state is more stable than the $\beta$-phase: the $\alpha \rightarrow \gamma$ bifurcation must occur at larger energies (smaller supercoolings) than the $\alpha \rightarrow \beta$ one, i.e.,

$$
e_{\alpha \gamma}>e_{\alpha \beta} .
$$

Comparing expressions (40) and (25) for a weak transformation, we obtain that the condition (41) is fulfilled for materials whose properties satisfy the criterion

$$
U \equiv \frac{C T_{E} B}{L^{2}}<\frac{1}{8} .
$$

Calculations, analogous to those that lead to Eq. (40), carried out for the $\beta$-phase, yield the expression for the bifurcational energy $e_{\gamma \beta} \approx-L-e_{\alpha \gamma}$, where the transition-state-line branches off of the homogeneous $\beta$-phase line, see Figs. 1 and 2 .

One should not forget about another possibility in the system that has been studied in the previous section, that is, decomposition into a heterogeneous mixture of $\alpha$ and $\beta$ phases. Therefore, the transition state, even if it is stable with respect to both homogeneous phases, may become unstable with respect to a heterogeneous state. To estimate this possibility one needs to apply the criterion (17) and (19) to the heterostate and find the state that branches off of the $\alpha$-phase first, as the energy of the latter decreases. To realize this idea we can employ a simplified capillary approach of Sec. III. Equating magnitudes of the threshold energies $e_{\alpha I}$ and $e_{\alpha \gamma}$, Eqs. (34) and (40) yields the expression for the critical thickness

$$
X_{\mathrm{cr}}=\frac{\sigma}{B} \text {. }
$$

Thus in plates thinner than $X_{\mathrm{cr}}$ made of a material with $U<1 / 8$, the parent phase must be replaced by the homogeneous transition state if the initial energy density of the system is in a certain band around the equaentropic point $e_{a \beta} \approx-L / 2$. In plates thicker than $X_{\mathrm{cr}}$ the transformation goes in the direction of the decomposition into a heterogeneous mixture of $\alpha$ and $\beta$ phases, see Figs. 1 and 2. Thus in small thermally insulated systems the homogeneous transition state becomes the most stable one which means that this state satisfies the definition of a phase. Such state may be called adiabatic nanophase. Evidently, mechanical, electrical and optical properties of such phase are different from those of bulk phases $\alpha$ and $\beta$. As it has been pointed out above, there may occur another bifurcation at still smaller energies after which a heterostate will gain the global stability.

The conditions of the adiabatic phase stabilization as represented by the critical magnitude of the parameter $U_{\text {cr }}=1 / 8$, see Eq. (42), and the critical thickness $X_{\text {cr }}$, Eq. (43), are the principal results of this work. However, thus far they were obtained with the help of an approximation $T_{\alpha \gamma}^{*}=T_{E}$, which may not hold in reality.To determine the conditions of adiabatic nanophase stabilization without unnecessary approximations one needs to apply more elaborate approach to this problem, which will be done below with the help of the continuum method.

\section{B. Continuum approach}

To address the issue of small particles many different theoretical methods can be used. In the present work we shall take advantage of the continuum method which allows one to study both equilibriumal and dynamical situations on the same ground. Within the framework of this method a thermodynamic system, in addition to temperature $T$ and pressure $P$, is characterized by another internal parameter $\eta$ which is a measure of disequilibrium in the system. At equilibrium the latter takes on a specific value which can be found from the proper thermodynamic condition and is a 
function of the local temperature and pressure: $\eta_{E}=\Phi(T, P)$. This parameter relates to the degree of a reaction in the theory of chemical reactions and macrokinetics, ${ }^{21}$ to the relaxation parameter in optics and theory of liquids, to the degree of nonrigidity in the cluster theory ${ }^{22}$ and magnetization in the theory of the magnetocaloric effect. ${ }^{23}$ In the theory of phase transitions it is associated with the symmetry change and is usually called order parameter. However, many transitions may have little to do with ordering, e.g., magnetic, polymorphic, freezing, spinodal decomposition. In what follows we shall call it transition parameter. Transition parameter is also a part of constitutive equations of materials, hence, making mechanical, electrical, magnetic, optical, etc. properties dependent on the transition parameter variations. The concept of the transition parameter helps one to simplify the definition of a phase which has been given in the beginning of the paper: homogeneous in the transition parameter and locally stable state of a thermodynamic system we shall call a phase.

In the framework of the continuum approach the problem of equilibrium and stability in isolated systems should be reformulated. If adiabatic conditions are maintained for a system then its energy $E$, which should be written as a functional, is a constant. If the system becomes heterogeneous there is a certain penalty on it which is expressed by the so-called gradient energy contribution into the functional of the total energy. ${ }^{24,25}$ Then

$$
E \equiv \int\left[e(\eta)+\frac{\kappa}{2}(\nabla \eta)^{2}\right] d^{3} x=\text { const, } \quad \kappa>0 .
$$

The integrand here represents the energy density of a heterogeneous system. There are no conservation constraints on the transition parameter because the latter is assumed to be nonconserved. However, the total volume, unlike the transition parameter, is conserved

$$
V \equiv \int d^{3} x=\text { const. }
$$

According to the principle (1) the entropy functional takes on a maximum value at the stable equilibrium state

$$
S \equiv \int s(e, \eta) d^{3} x \rightarrow \text { maximum. }
$$

Here the entropy functional $S$ is assumed not to contain the gradient term. The entropy $s(e, \eta)$ and energy $e(\eta)$ densities of a homogeneous system can be found if the free energy of a unit volume of a material is known as a function of temperature and transition parameter, $f(T, \eta)$. For this one needs to use the equilibrium Legendre transform with respect to temperature because the transition parameter is not involved in it

$$
s(e, \eta)=-\frac{\partial f(T, \eta)}{\partial T} ; \quad f(T, \eta)=e(\eta)-T s(e, \eta)
$$

Thus in the framework of a continuum theory equilibrium states of an isolated system obey the conditions of the isoperimetric problem in the calculus of variations, Eqs.
(44)-(46). The properties of these states have been studied extensively in Ref. 12 and will be reviewed here briefly. The equilibrium states fall into two categories: those which allow Lagrange multipliers and those which do not. ${ }^{26}$ The latter states are inhomogeneous both in temperature and transition parameter distributions. They may be realized in materials with the vanishing thermal conductivity only ${ }^{12}$ and will not be considered any further. The former states have uniform distribution of temperature $T=(\delta S / \delta E)^{-1}$ across the system and benefit from the introduction of the free energy functional

$$
F \equiv \int\left[f(T, \eta)+\frac{\kappa}{2}(\nabla \eta)^{2}\right] d^{3} x .
$$

These are the same states as those of the isothermal system and may be represented by solutions of the Euler equation

$$
\frac{\grave{o} f(T, \eta)}{\grave{o} \eta}-\kappa \nabla^{2} \eta=0, \quad T=\text { const. }
$$

As the boundaries of the system in question do not interact with the environment (free surface), the variational problem yields the Neumann-type boundary conditions

$$
\partial_{n} \eta=0 \text { at the surface. }
$$

\section{Local stability of equiliubrium states}

The free energy density of a system capable of undergoing a phase transition $f(T, \eta)$ is a continuous function of the transition parameter. Homogeneous solutions of Eq. (49) $\eta=\eta_{E}(T)$, that is those with $\nabla \eta=0$, correspond to the maxima and minima of the free energy density $f(T, \eta)$ as a function of the transition parameter.

To address a problem of phase transformations of the first order we must assume that $f(T, \eta)$ has at least two minima associated with the $\alpha$ and $\beta$ phases. ${ }^{27}$ At the equilibrium temperature, transition parameter takes on the values $\eta_{\alpha}$ and $\eta_{\beta}$ in the $\alpha$ and $\beta$ phases, respectively, and the free energy densities of these states are equal [see Eq. (10) and curve 2 in Fig. 4]. Since two minima of a continuous function must be separated by a maximum, it follows that the transition state $\gamma$ corresponds to the free energy maximum (saddle in a multidimentional case) whose position between the bulk phases may vary with temperature

$$
\frac{d \eta_{\gamma}}{d T}=-\frac{f_{\eta T}\left(T, \eta_{\gamma}\right)}{f_{\eta \eta}\left(T, \eta_{\gamma}\right)} .
$$

To ensure local stability under isothermal conditions (open system), the isothermal "stiffness" of the equilibrium state should be positive: $f_{\eta \eta}\left(T, \eta_{E}\right)>0$. This is true for $\alpha$ and $\beta$ phases: $f_{\eta \eta}\left(T, \eta_{\alpha, \beta}\right)>0$. The isothermal stiffness of the transition state is negative: $f_{\eta \eta}\left(T, \eta_{\gamma}\right)<0$, Fig. 4. This means that this state does not correspond to any metastable bulk phase of an open system. However, constraint of isolation changes the condition of local stability of this states ${ }^{11,12}$ and isothermally unstable state $\eta=\eta_{E}$ will be thermodynamically stable if the adiabatic stiffness of this state is posi- 


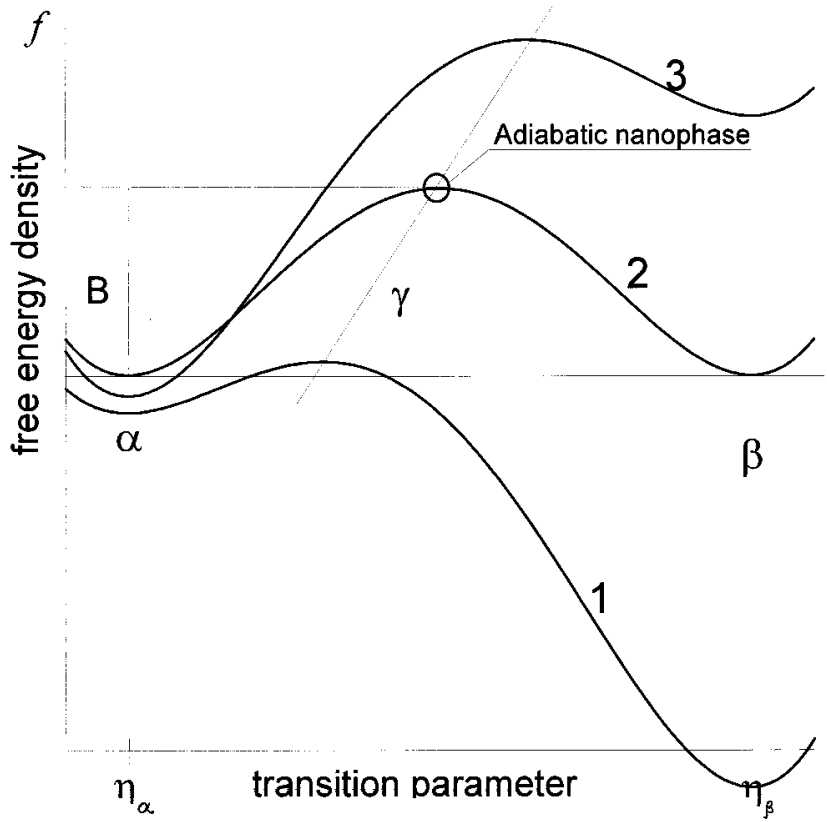

FIG. 4. Free energy density of the system with $Q=0.1$ and $\Sigma=0.009$ as a function of the transition parameter at different temperatures: (1) $T<T_{E}$; (2) $T=T_{E}$; (3) $T>T_{E}$.

tive: $f_{\eta \eta}-\left(f_{\eta T}\right)^{2} / f_{T T} \geqslant 0$. Hence, an isothermally unstable transition state $\gamma$ will be adiabatically stable if the modulus $M$ takes on a value greater than one

$$
M \equiv \frac{f_{\eta T}^{2}\left(T, \eta_{\gamma}\right)}{f_{\eta \eta}\left(T, \eta_{\gamma}\right) f_{T T}\left(T, \eta_{\gamma}\right)}>1 .
$$

It is of interest to calculate the specific heat of the transition state. Substituting Eq. (51) into the definition (14) and taking into account that $C=(\partial e / \partial T)_{\eta}$ we arrive at the expression for the specific heat of the transition state

$$
C_{\gamma} \equiv \frac{d e\left(\eta_{\gamma}\right)}{d T}=\left(\frac{\partial e}{\partial T}\right)_{\eta}+\left(\frac{\partial e}{\partial \eta}\right)_{T} \frac{d \eta_{\gamma}}{d T}=C(1-M) .
$$

This expression shows that when the transition state gains adiabatic stability, its specific heat becomes negative which means that such a state would be unstable in the bulk. With regard to the entropy-versus-energy function of the transition state $s_{\gamma}(e)$, it is necessary to stress out that its plot ceases to be convex upward as soon as the state becomes stable, see Eq. (14) and Fig. 1.

\section{Global stability of equiliubrium states}

Besides homogeneous solutions that correspond to the bulk phases $\alpha, \beta$ and the transition state $\gamma$, Eq. (49) is known to have bounded heterogeneous equilibrium solutions that correspond to the transition regions where transition parameter varies rapidly from the value on one side to the value on the other side. In a 1D system Eq. (49) may be integrated once to yield

$$
\frac{\kappa}{2}\left(\frac{d \eta}{d x}\right)^{2}=\Delta f(T, \eta)-G ; \quad \Delta f \equiv f(T, \eta)-f_{\alpha}(T),
$$

where $\Delta f(T, \eta)$ is the excess free energy density of the system. As a consequence of the no-flux boundary conditions (50), the integration constant $G$ vanishes in the thermodynamic limit but is not zero in a finite size system and has a meaning of the excess free energy density on the surface

$$
G=\Delta f\left(T, \eta_{1}\right)=\Delta f\left(T, \eta_{2}\right) .
$$

Transition parameter values on the surface of a heterogeneous layer of finite thickness, $\eta_{1}$ and $\eta_{2}$, differ from bulk values, $\eta_{\alpha}$ and $\eta_{\beta}$, and generate the surfacial ordering $\left(\eta_{1}-\eta_{\alpha}\right)$ and disordering $\left(\eta_{\beta}-\eta_{2}\right)$, respectively.

General solution of Eq. (54) may be represented in the form of Elliptic integral and is a vast subject in its own right: it is either monotonic-interface, or periodic_-domains and interfaces. The latter are not considered in the present study because they may be locally stable at most. Qualitative properties of the monotonic equilibrium heterostate (interface) can be derived from the analysis of the bifurcational map

$$
X=\sqrt{\frac{\kappa}{2}} \int_{\eta_{1}}^{\eta_{2}} \frac{d \eta}{\sqrt{\Delta f(\eta, T)-G}} .
$$

Also Eq. (54) and boundary condition (55) help find the expressions for the surface energy and thickness of the interface. Adopting the definitions of these quantities introduced in Ref. 25, we obtain

$$
\sigma(T, X) \equiv \int_{0}^{X} \kappa(\nabla \eta)^{2} d x ; \quad l_{I}(T, X) \equiv \frac{\eta_{2}-\eta_{1}}{\max |\nabla \eta|} .
$$

There is an apparent analogy between equilibrium problem, as represented by Eqs. (54) and (55), and mechanical problem of oscillations of a point mass $\kappa$ with the speed $d \eta / d x$ in the potential field $\Pi(\eta)=-\Delta f(T, \eta)$, where $\eta_{\gamma}$ corresponds to the bottom of the potential well. Equation (54) manifests conservation of the mechanical energy, $\eta_{1}$ and $\eta_{2}$ have the meaning of the turning points, the thickness of the layer $X$ is analogous to the period of mechanical oscillations, interfacial thickness - to the time constant and the surface energy - to the total action of the system. As is well known, there impossible oscillations in such system faster than harmonic ones, that is with small amplitude.

In a very thin layer the surfacial (dis)ordering becomes very strong and the transition parameter distribution approaches the homogeneous magnitude of the transition state parameter- $\eta_{\gamma}$. Equation (43) gives an approximate value of the critical thickness $X_{\mathrm{cr}}$ such that in thinner plates the homogeneous transition state is the most stable one (adiabatic nanophase). The exact criterion of the global stability of the transition state against a heterostate may be derived from the analysis of the bifurcational map (56). The mechanical analogy suggests that in a small vicinity of the transition state a heterogeneous solution of Eq. (54) may be expressed as a small harmonic modulation $\delta \eta$ of the amplitude $H$ on the top of a homogeneous transition state $\eta_{\gamma}$. Then the criterion of bifurcation of such a solution from the homogeneous one may be found from Eq. (56) as 


$$
\begin{aligned}
\widetilde{X} & =\sqrt{\frac{\kappa}{\left|f_{\eta \eta}\left(T, \eta_{\gamma}\right)\right|}} \int_{-H}^{H} \frac{d(\delta \eta)}{\sqrt{H^{2}-(\delta \eta)^{2}}} \\
& =\pi \sqrt{\frac{\kappa}{\left|f_{\eta \eta}\left(T, \eta_{\gamma}\right)\right|}} .
\end{aligned}
$$

\section{E. Adiabatic nanophase stability}

To advance in the analysis of the adiabatic nanophase stability it is desirable to have a comprehensive picture of the free energy which reflects microscopic interactions in the system and may be employed for the continuum approach. Then Eqs. (17)-(20) and (58) will help us refine the rough estimates of the adiabatic nanophase-stability limits, made in the beginning of this Section, based on the assumption of the equilibrium temperature of the transition state. An important question should be asked: How one can determine parameters of the free energy? One way to solve this problem (macroscopic) is to obtain expressions for various measurables and compare them with the experimental observations of kinetics and equilibria in two-phase systems. Another way (microscopic) consists in deriving parameters of the mesoscopic free energy from an appropriate microscopic theory, e.g., transition state theory, where the isothermal stiffnesses $f_{\eta \eta}\left(T_{E}, \eta_{\alpha, \beta}\right)$ relate to the frequencies of intrawell vibration while $\left|f_{\eta \eta}\left(T_{E}, \eta_{\gamma}\right)\right|$ relates to the interwell motion. Importantly to realize that the free energy might not necessarily be symmetric with respect to the $\eta_{\alpha}$ and $\eta_{\beta}$ phases even at the equilibrium temperature. Such asymmetry manifests in the difference between the isothermal stiffnesses of the equilibrium states as well as in the different distances from the transition-state parameter $\eta_{\gamma}\left(T_{E}\right)$ to that of the equilibrium states $\alpha$ and $\beta$. A strong indication of the asymmetry of the free energy of the melting-freezing transition is a possibility to supercool liquids for up to 100 degrees and virtual impossibility to superheat solids at all. This may embody a problem for the modeling of the free energy potentials by the polynomials of low order because the polynomial of the fourth order, which is the lowest order polynomial to exhibit the "double-well" shape, has equal second derivatives in both minima. Thus polynomials may be used for modeling the first order transitions close to the second order and are not appropriate functions for modeling the strongly first order transitions like melting-freezing.

Many robust characteristic features of transformations can be drawn from properties of the transition state only. Therefore, we shall not deepen into the study of analytical functions appropriate for the purposes of the phase transition modeling and we shall not consider effects associated with the difference of isothermal stiffnesses of $\alpha$ and $\beta$ phases. Instead, for the excess free energy $\Delta f$ we will use the expression

$$
\begin{aligned}
& \Delta f(T, \eta)=\frac{B}{2^{4}} \eta^{2}\left[h-\frac{2}{3}(h+2) \eta+\eta^{2}\right], \\
& h(T) \equiv 1+3 \frac{2^{4}}{\Sigma} \frac{T-T_{E}}{T_{E}},
\end{aligned}
$$

where $\Sigma \equiv B / L$ is the ratio of two basic scales of the free energy. The transition parameters of $\alpha$ and $\beta$ phases remain unchanged with temperature for this potential: $\eta_{\alpha}=0$ and $\eta_{\beta}=1$, and the location of the transition state depends upon the normalized temperature $: \eta_{\gamma}=\frac{1}{2} h(T)$. This potential, see Fig. 4, has been adopted from Refs. 12 and 28 where all its properties may be found.

To apply the free energy (59) to the problem of adiabatic phase stability, as expressed by the bifurcation criterion (17) and (19), one has to calculate the excess free $\Delta f_{\gamma}$ and internal $\Delta e_{\gamma}$ energies of the transition state as functions of its temperature and substitute these into Eq. (19). The free energy (59) yields $\Delta f_{\gamma}=1 / 3 B h^{3}(4-h)$ and $\Delta e_{\gamma}=$ $-1 / 4 L h^{2}\left[3-h-4 \Sigma(2-h)^{2}\right]$ and Eq. (19) takes the form:

$$
U=\frac{9}{32} h_{\alpha \gamma}^{*} \frac{\left[3-h_{\alpha \gamma}^{*}-\Sigma\left(2-h_{\alpha \gamma}^{*}\right)^{2}\right]^{2}}{\left(4-h_{\alpha \gamma}^{*}\right)\left[3-4 \Sigma\left(1-h_{\alpha \gamma}^{*}\right)\right]} .
$$

The solution of this equation enables us to find the bifurcation temperature $T_{\alpha \gamma}^{*}$ and energy $e_{\alpha \gamma}=C\left(T_{\alpha \gamma}^{*}-T_{E}\right)+\Delta e_{\gamma}\left(h_{\alpha \gamma}^{*}\right)$ and represent the bifurcational criterion (41) as follows:

$$
U<\frac{3}{32} \frac{1-\frac{1}{2}\left(h_{\alpha \gamma}^{*}\right)^{2}\left[3-h_{\alpha \gamma}^{*}-\Sigma\left(2-h_{\alpha \gamma}^{*}\right)^{2}\right]}{1-h_{\alpha \gamma}^{*}} .
$$

Equation (60) has a solution between the congruent points $h_{1}=0$ and $h_{2}=2$ only if $U<0.12$. For small values of $U$ this solution takes the form

$$
h_{\alpha \gamma}^{*} \approx \frac{128}{27} U ; \quad e_{\alpha \gamma}=-\frac{16}{3} L U\left(1-\frac{128}{81} U\right) .
$$

Then the criterion (61) alows us to conclude that the adiabatic nanophase is possible for materials with parameters such that:

$$
U \leqslant U_{\mathrm{cr}} \equiv \frac{3}{32} \approx 0.0938
$$

To determine the temperature of the local stability boundary $T_{\gamma}$ of the transition state, as expressed by the inequality (52), one needs to calculate the modulus $M$ and equate it to one: $M=(9 / 64 U) h_{\gamma}\left(2-h_{\gamma}\right)$ $\times\left[1-(16 / 3) \Sigma\left(1-h_{\gamma}\right)\right]=1$. Solving this equation one can find the normalized temperature and energy of the local stability boundary

$$
h_{\gamma} \approx \frac{32}{9} U, \quad e_{\gamma} \approx-\frac{16}{3} L U\left(1-\frac{16}{9} U\right) .
$$

Comparing Eqs. (62) and (64) one finds that the temperature of the local stability boundary is less than that of the global stability and both are less than the equilibrium temperature, i.e., $T_{\gamma} \leqslant T_{\alpha \gamma}^{*}<T_{E}$. However, the energy of the local stability boundary is greater than that of the global stability because the specific heat of the transition state is negative [see Eqs. (52) and (53) and Insert in Fig 1]. Hence, the energy band of the global stability of the transition state is narrower than that of the local stability, with the transition state being 
more stable than both homogeneous phases $\alpha$ and $\beta$ in the energy band $e_{\gamma \beta}<e<e_{\alpha \gamma}$, where $e_{\gamma \beta} \approx-L-e_{\alpha \gamma}$.

However, as we discussed above, after transformation the layer may turn heterogeneous if its thickness is greater than $\tilde{X}$, Eq. (58). The free energy (59) allows one to calculate the value of the bifurcational thickness and relate it implicitly to the initial energy density $e^{0}=e_{\gamma}(h)$ $=C\left(T-T_{E}\right)+\Delta e_{\gamma}(h)$

$$
\widetilde{X}=\frac{X_{\mathrm{cr}}}{\sqrt{h(2-h)}} ; \quad X_{\mathrm{cr}}=\frac{\pi}{4} \sqrt{\frac{\kappa}{B}} .
$$

To reveal the inherent nature of the critical thickness (65) we need to analyze the properties of the phaseseparating interface with the surface energy and thickness as the most important ones. The free energy (59) allows us to determine the surface energy and thickness of the interface between $\alpha$ and $\beta$ phases, Eqs. (57), in the thermodynamic limit

$$
\sigma_{\infty}=\frac{2}{3} \sqrt{2 \kappa B}, \quad l_{\infty}=\frac{1}{4} \sqrt{\frac{\kappa}{2 B}} .
$$

Comparing the formulae (65) and (66) one can see that $X_{\mathrm{cr}}=\sqrt{ } 2 \pi l_{\infty}$, i.e., the critical thickness is approximately equal five times the interfacial thickness. The bifurcational size $\widetilde{X}$, below which the adiabatic nanophase appears on the phase diagram, depends upon the initial energy $e^{0}$ (supercooling $\Delta \theta$ ) and may exceed $l_{\infty}$ up to 20 times (Fig. 2). In Sec. III we found that the heterostate zone on the phase diagram $(e, X)$ is separated from the single phase zones $\alpha$ and $\beta$ by two branches of the curve $X^{*}(e)$, Eqs. (34) and (35) and Fig. 2. Merging of $X^{*}(e)$ and $\widetilde{X}(e)$ creates two "triple points:" $\operatorname{Tr}_{\alpha}$ and $\operatorname{Tr}_{\beta}$. Although behavior of the phase boundary in the vicinity of these points is not known to the author at present, he believes that, in fact, $X^{*}(e)$ and $\widetilde{X}(e)$ represent one smooth curve that separates one-phase and two-phase zones on the phase diagram. Also Eqs. (34), (38), (63), and (66) prove that the adiabatic nanophase existence imposes the restriction on the hierarchy of length scales in the system

$$
l_{A} \ll l_{C} \approx l_{\infty} \text {. }
$$

Expressions of $U_{\mathrm{cr}}$, Eq. (63), and the critical thickness $X_{\text {cr }}$, Eq. (65), derived with the help of the mesoscopic free energy (59), refine the values of these parameters, Eq. (42) and (43), estimated on the premise of the equilibrium temperature of the $\gamma$ state after transition and demonstrate that the latter assumption is reasonable. Eq. (39), for instance, reveals the nature of the $\alpha \rightarrow \gamma$ transition as a weakly first order because there is a small jump between temperatures of the phases, that is inverse derivatives of entropy with respect to energy. At the same time, the continuum method allows one to arrange the bifurcational energies on the phase diagram of a system with small $U$ in the ascending order: $e_{\beta}^{E}<e_{\gamma \beta}<e_{\alpha \beta}<e_{\alpha \gamma}<e_{\gamma}<e_{\alpha}^{E}$, and to identify the region of stability of the adiabatic nanophase on the phase diagram $(e, X)$ as the box of the width $L\left[1-\left(U / U_{\mathrm{cr}}\right)+(4 / 27)\right.$ $\left.\times\left(U / U_{\mathrm{cr}}\right)^{2}\right]$ around the center of the two-phase zone (equaentropic point) and the height $X_{\text {cr }}$ which is inversely proportional to the square root of $B$, Figs. 1 and 2 .

\section{COMPARISON WITH OTHER STUDIES}

Umantsev and Olson ${ }^{28}$ carried out the numerical simulation of transformation dynamics in layers of materials with different parameters $U$, thicknesses $X$, and initial supercoolings $\Delta \theta$. The authors found that in the thin layer of the thickness $1.125 X_{\text {cr }}$ (line 3 in Fig. 2) the transition state appeared and did not decompose for supercoolings $0.25<\Delta \theta<0.285$, although in a large system of $X \approx 100 X_{\text {cr }}$ with the same parameters the ultimate equilibrium had been attained on the heterogeneous mixture of $\alpha$ and $\beta$ phases. For larger values of $\Delta \theta$ the equilibrium distribution of the transition parameter in a thin layer appeared to be modulated by a harmonic wave of a small amplitude. This type of the dynamic behavior supports the inference that the transition state may be the most stable one when in confinement. In Ref. 29 the authors analyzed the linear dynamic stability of homogeneous equilibrium states in 3D systems of arbitrary shape. The analysis revealed that such a state is linearly stable if the following two conditions are simultaneously satisfied:

$$
\begin{array}{cc}
f_{\eta \eta}-\left(f_{\eta T}\right)^{2} / f_{T T} \geqslant 0 & \text { for }|\mathbf{k}|=0 \\
\text { and } & \\
f_{\eta \eta}+\kappa \mathbf{k}^{2} \geqslant 0 & \text { for }|\mathbf{k}| \neq 0,
\end{array}
$$

where $\mathbf{k}$ is the wave vector of the permitted perturbations. In the thermodynamic limit of an infinite system all perturbations, including long range ones with $|\mathbf{k}| \rightarrow 0^{+}$, are permissible. This yields the criterion $f_{\eta \eta} \geqslant 0$ which is fulfilled for states $\alpha, \beta$ and is not fulfilled for the transition state $\gamma$. However, this is not so in a confinement where the permitted wavevectors $\mathbf{k}$ of heterogeneous modes constitute a discrete set with the limited from below absolute value $|\mathbf{k}|$. Likewise, confinement of traveling waves entails standing waves with quantized frequencies. The quantization of permitted wavevectors stabilizes the transition state $\gamma$. In a 1D layer with the boundary conditions (50) $\min |\mathbf{k}|=\pi / X$ and the criterion of linear stability (68) for the transition state takes the form that coincides completely with Eqs. (52) and (58) which have been derived from the thermodynamical principle of the global stability (1). Combination of thermodynamic and dynamic stability analyses compels us to believe that the globally stable transition state is a truly equilibrium phase and not an artifact. It comes about as a result of two mutually assisting factors: adiabatic insulation and confinement, and inequalities (68) are the conditions for the existence of the adiabatic nanophase.

Molecular dynamics have been extensively used for the analysis of structural evolution of clusters (small particles) at constant energy. When molecular dynamics is used to analyze the behavior of materials, one has to be careful while interpreting computer experiments because they are usually done under systems of finite size and constant energy. Rose and Berry ${ }^{30}$ studied melting and freezing of small salt clusters $(\mathrm{K}-\mathrm{Cl})$. They found that in a certain energy band, except 
solid-like and liquid-like, other states that correspond to the saddle points of the potential energy surface are possible. In some experiments the "equilibrium" has been achieved on "fluctuating states." Similar results were observed earlier by Sugano and Savada in the six-particle transition-metal clusters. ${ }^{31}$

Suzuki and Takahashi ${ }^{32}$ studied the nucleation mechanism for the martensitic transformation in the crystal composed of particles interacting with 12-6 and 8-4 LenardJones potentials. They observed that the bcc lattice always transformed through a martensitic transformation into one of the mechanically stable, fcc or hcp, structures when in large volumes or even in small volumes but with the 12-6 potential. However, the 8-4 type lattice did not exhibit a martensitic transformation and remained bcc when confined to a small volume of the order of nanometers. This stabilization mechanism, related to confining geometries, remained unexplained in the paper and may be understood on the basis of the present treatment. Mechanical instability of the bcc lattice occurs because the $\left(C_{11}-C_{12}\right)$ elastic stiffness of this structure is negative for both types of potentials. ${ }^{32,33}$ As strain is the transition parameter for martensitic transformations, the elastic stiffness is $f_{\eta \eta}$ and the bcc structure may be interpreted as the transition state between two stable configurations. In addition, mechanical calculations of Ref. 33 show that the absolute value of the 12-6 type elastic stiffness is approximately seven times greater than that of the 8-4 lattice. Although not stated explicitly, the numerical experiments were conducted apparently under adiabatic conditions. Therefore, the homogeneous stability of the lattice instead of the isothermal stiffness $f_{\eta \eta}$ is determined by the adiabatic one $f_{\eta \eta}-\left(f_{\eta T}\right)^{2} / f_{T T}$ which is more positive than the former. Notice that the second term here is proportional to the thermal expansion coefficient squared. ${ }^{34}$ Of course, lattice stability can be infringed not only by homogeneous distortions but by heterogeneous ones also. All this has led us to the second condition of stability in confined geometries: $f_{\eta \eta}+\kappa(\pi / X)^{2} \geqslant 0$. Apparently, both conditions are satisfied for small particles of the 8-4 type lattice, despite its isothermal mechanical instability, but the second condition is not satisfied even for the smallest sizes of the 12-6 type lattice because the isothermal stiffness $f_{\eta \eta}$ of this lattice is "very negative."

Cheyssac, Kofman and Garrigos ${ }^{35}$ optically investigated solid-liquid phase transition in lead aggregates of very small sizes (from 23 to $300 \AA$ ) and found a huge hysteresis in the reflectance-versus-temperature curve which meant that there was a wide gap between temperatures of melting and freezing of aggregates. The isothermal size effect may account only for the melting temperature decrease while strong supercooling of the liquid phase below the melting temperature should be explained by the adiabatic effect [see Eq. (34), the subsequent discussion and Eq. (36)]. Appearance of the adiabatic nanophase may be responsible for the disappearance of the hysteresis for the lower sizes.

Kim, Lin and Kelly ${ }^{36}$ studied solidification of submicron droplets, $10-60 \mathrm{~nm}$, of high purity elemental metals by electrohydrodynamic atomization in vacuum when droplets so- lidify in the free flight. They found that under extreme conditions of high cooling rates and very small liquid volumes some pure metals solidified from the melt as an amorphous phase. Also they found that the critical size increased with decreasing melting temperature of each bcc metal except iron and that the glass transition temperature increased with the latent heat of fusion. The combination of high cooling rates and small volumes of particles as necessary conditions for amorphization conjectures that the amorphous phase may be identified with the adiabatic nanophase considered in the present paper. A possibility for a material to have adiabatic phase is determined by the magnitude of the parameter $U$, Eqs. (42) and (63). This may explain why some pure metals do exhibit amorphous states and some don't. The maximum temperature of the parent phase (liquid) when the transformation into adiabatic phase becomes possible $T_{\alpha \gamma}^{0}$, Eq. (39), may be associated with the glass transition temperature. The support for the adiabatic theory of amorphization also comes from the fact that the glass transition temperature increases together with the latent heat. ${ }^{36}$ More details on the adiabatic theory of amorphization will be presented elsewhere.

Experimental estimates of the critical size $X_{\mathrm{cr}}$, Eq. (43), may be used for the determination of the activation barrier of transition if the surface energy is known from independent measurements. For the solid-liquid transition in simple metals, $X_{\text {cr }}$ ranges from few to tens of nanometers. ${ }^{35,36}$ Taking $100 \mathrm{ergs} / \mathrm{cm}^{2}$ as a typical value of the solid-liquid surface tension, we estimate the activation barrier $B$ as $10^{7} \mathrm{~J} / \mathrm{m}^{3}$. Unfortunately, independent estimates or direct measurements of this quantity are not known to the author although it is important for the nucleation theory.

Above described results of numerical and physical experiments suggest that the constraint of energy conservation stabilizes saddle-point configurations and provide a reasonable proof for the adiabatic nanophase appearance after transformations. It may be advantageous to try in the future to conduct experiments under conditions of adiabatic insulation instead of isothermal ones.

\section{DISCUSSION}

In this work a one-component system capable of undergoing a first-order phase transition was analyzed under conditions of thermal insulation. The functional space even of the simplest possible system without spinodal or critical points and with only two bulk phases, $\alpha$ and $\beta$, contains different states which may be at least locally stable at appropriate energies. Among most important ones for the present discussion should be named the heterogeneous state where $\alpha$ and $\beta$ phases are physically separated by the interface and the homogeneous transition state $\gamma$ which separates $\alpha$ from $\beta$ in the functional space. Transformation fraction $\varphi$ is the most convenient quantitative characteristic of a heterostate, while the transition parameter $\eta$ characterizes the homogeneous state. To determine the most stable state we used the Clausius-Gibbs criterion (1) of the global stability which may be expressed in the form of the general criterion of bifurcation of various states. The main hardness of the global 
stability analysis stems from the fact that the states to be compared do not occupy neighboring volumes in the phasespace of the system. The global stability criterion takes the form of Eqs. (17) and (19) if the excess quantities of the state satisfy inequality (20). Unlike an open (isothermal) system where the global optimizer in the thermodynamic limit is a completely structureless (homogeneous) state, in a closed (adiabatic) system the global optimizer may have a structure, that is may be a heterogeneous mixture of coexisting phases $\alpha$ and $\beta$, if the average energy of the system belongs to a certain band: $e_{\beta}^{E}<e<e_{\alpha}^{E}$. Importantly that in adiabatic system the global stability may be achieved on the state that does not have minimum free energy at that temperature. Indeed, the surface energy $\sigma A$ of a heterostate is the free energy surplus which would have been absent in a homogeneous $\alpha$ or $\beta$ phase at the equilibrium temperature. Notice that the latter possesses a certain degree of "stability" in the sense that, in the energy band of phase coexistence, temperature of a system tends to the equilibrium value regardless of its initial magnitude. In Fig. 1 entropies of different locally stable equilibrium states of an adiabatic system are depicted as functions of their energies. The lines of bulk phases $\alpha$ and $\beta$ intersect at the equaentropic point $e_{\alpha \beta}$. It is advantageous for a system to create an internal interface separating two stable phases $\alpha$ and $\beta$ in the energy band $e_{\beta}^{E}<e<e_{\alpha}^{E}$ which includes the equaentropic point. The straight line 1 in Fig. 1, which is tangential to both single phase curves, represents the $\alpha+\beta$ heterostate, Eq. (12). The points of tangency $e_{\alpha}^{E}$ and $e_{\beta}^{E}$ and the equaentropic point $e_{\alpha \beta}$ break the energy axes down into four different regions where the parent phase either transforms completely into phase $\beta$ ( $e<e_{\beta}^{E}$, "massive", transformation), or $\left(e_{\beta}^{E}<e<e_{\alpha}^{E}\right)$ decomposes into a twophase heterostate of $\alpha$ and $\beta$ phases with fixed energies, $e_{\alpha}^{E}$ and $e_{\beta}^{E}$, respectively, and the relative proportions given by the lever rule (13). For the average energy density above $e_{\alpha}^{E}$ the transformation does not go at all and the system stays in the parent phase. In the energy band $e_{\beta}^{E}<e<e_{\alpha \beta}$ the transformation path passes through the metastable homogeneous state $\beta$ of the temperature above equilibriumal, and the system may be trapped there (heat-trapping). The real scenario of transformation depends upon thermodynamic and kinetic parameters of the system. ${ }^{37}$ For instance, if parameter $U$ is small enough, $U \ll 1$, the $\alpha \rightarrow \beta$ transformation takes a path of continuous modulations wich is directly analogous to the spinodal decomposition when the system generates many interfaces at the beginning and slowly eliminates them later (coarsening). On the other hand, if $U$ is large enough, $U \gg 1$, the transformation follows a traditional path of nucleation and growth with the hybrid mode possible for moderate $U \gtrsim 1$.

The transformation in a closed system of finite size does not start immediately after supercooling of $\alpha$ phase below the equilibrium temperature but is deferred until greater magnitudes of supercoolings. The main reason of existence of the threshold energy $e_{\alpha I}$ is the necessity to accommodate an interface which possesses the interfacial energy. In the energy band $e_{\beta I}(X)<e<e_{\alpha I}(X)$, Eqs. (34) and (35), temperature of the heterostate equals equilibriumal which means that the free energy of this state is greater on the amount $\Delta F=\sigma A$ than that of homogeneous phases $\alpha$ or $\beta$ at the same temperature. On the Mollier chart (Fig. 1) the heterostate of a finite-size system is represented by the straight line 2 which lies below that of the infinite system. An important finding of this study is the revelation of a strong size dependence $\left(\propto X^{-1 / 2}\right)$ of the adiabatic phase diagram which is not the case in the isothermal system where the size-effect is due to the surfacial interactions exclusively.

However, the most striking feature of an adiabatic system is the stabilization of the transition state in very small particles of materials with $U<U_{\mathrm{cr}}$. The beauty of this result is the fact that the transition state possesses maximum free energy among all other homogeneous states of the system at the same temperature. This result suggests that all transitions may be classified with respect to the value of the parameter $U$. The transition state $\gamma$ is locally stable in the energy band where the adiabatic "stiffness" is positive and the $\gamma$-state line in this domain is convex downward, see Eq. (52) and in Fig. 1. The global stability of this state spreads over slightly narrower energy band $\left(e_{\beta \gamma}, e_{\gamma \alpha}\right)$, Eqs. (62) and (64), where the end points are the intersections of the $\gamma$-state line with the $\beta$ and $\alpha$ bulk-phase-lines respectively (Fig. 1). The general criterion of stability (17)-(20), demonstrates that the $\alpha \rightarrow \gamma$ transition is weakly first-order because there is a small jump of the derivative of entropy with respect to energy, that is inverse temparature. The thermodynamic stability analysis demonstrate that there exists the critical thickness $X_{\mathrm{cr}}$ such that in layers of thickness less than the critical $X<X_{\text {cr }}$ creation of a phase-separating interface is not favorable and the transition state becomes the global optimizer-adiabatic nanophase. Linear dynamic stability analysis confirms the inference of the absolute stability of such a phase in layers of thickness $X<X_{\mathrm{cr}}$. This makes the activated configuration globally stable in this energy band not only with respect to the bulk phases but with respect to the heterostate also.

The bifurcational scenario is different in systems of different sizes and actually may be very peculiar. As energy density of a particle with the linear size that corresponds to the dashed line 3 in Fig. 2 decreases below $e_{\alpha \gamma}$, the parent phase transforms into the adiabatic phase. Further reduction of energy leads to the appearance of heterogeneity in the system which will be replaced by the adiabatic phase again if energy is decreased even further. In this window of instability a heterogeneous structure of a particle consists of regions occupied by $\alpha$-like and $\beta$-like phases with relative proportion dependent on the energy of the particle. The last bifurcation occurs at $e_{\beta \gamma}$ when the adiabatic phase transforms into the homogeneous $\beta$-phase.The bifurcational size $\widetilde{X}$ depends slightly upon energy of the system and may span from few to 20 interfacial widths [Eq. (65) and Fig. 2]. Stabilization of the transition state comes about as a result of two mutually assisting constraints: insulation and confinement. In small 3D spheroidal or cuboidal particles this effect is enhanced by the dimensionality of the system as compared to the 1D layers considered in the present study. It is completely equilibriumal effect which has nothing to do with kinetics in confinement. Notice that, while heat transfer outside of the 
system is not permitted, the thermal conductivity inside the system is normal. In Sec. I we explained that before the transition the system should be prepared in the supercooled state and isolated from the environment then. If one would open the system up after the transition to adiabatic phase and expose it to the heat exchange with a thermal reservoir of the same temperature, it will destroy the delicate balance of the adiabatic nanophase and shift the equilibrium in the direction of $\alpha$ or $\beta$ phase. This property of adiabatic nanophase allows one to use such materials as sensors of insulation.

In case of small particles of solid solution, where mass conservation replaces the conservation of energy, present result predicts the appearance of the new stable phase with composition deeply inside the miscibility gap. This phase should occur at the normalized supersaturation about 0.5 . To conclude, we can say that dispersion of particles in composite materials is a very efficient way to alter their properties and has a strong potential in the creation of new multiphase smart materials.

${ }^{1}$ R. Clausius, Ann. Phys. 125, S. 400 (1865).

${ }^{2}$ J. W. Gibbs, The Scientific Papers (Dover, New York, 1961), Vol. 1.

${ }^{3}$ M. Planck, Thermodynamik, 3rd ed. (Veit, Leipzig, 1911), p. 279.

${ }^{4}$ E. A. Guggenheim, Thermodynamics (North-Holland, Elsevier Science, Amsterdam, 1967).

${ }^{5}$ J. Kestin, A Course in Thermodynamics (Blaisdell, Waltham, 1966), p. 513.

${ }^{6} \mathrm{H}$. B. Callen, Thermodynamics and Introduction to Thermostatics (Wiley, New York, 1985).

${ }^{7}$ G. Weinriech, Fundamental Thermodynamics (Addison-Wesley, Reading, 1968), p. 162.

${ }^{8}$ M. E. Glicksman and R. J. Shaefer, J. Cryst. Grow. 1, 297 (1967).

${ }^{9}$ E. Meyer, J. Cryst. Grow. 74, 425 (1986); in Current Topics in Crystal Growth Research, Vol. 2, edited by J. Menon (Research Trends, Trivondrum, India, 1995), p. 123.

${ }^{10}$ J. Schmelzer and H. Ulbricht, J. Colloid Interface Sci. 128, 104 (1989).
${ }^{11}$ L. Stodolsky, Phys. Res. A 39, 3646 (1989).

${ }^{12}$ A. Umantsev, J. Chem. Phys. 96, 605 (1992).

${ }^{13}$ L. D. Landay and E. M. Lifshitz, Statistical Physics (Pergamon, Oxford, 1958).

${ }^{14}$ P. A. Thompson and G. E. A. Meier, in PDEs and Continuum Models of Phase Transitions (Proceedings of an NSF-CNRS Joint Seminar), edited by. M. Rascle, D. Serre and M. Slemrod; (Springer, Berlin, 1989), p.146.

${ }^{15}$ M. I. Kaganov and A. N. Omel'yanchuk, Sov. Phys. JETP 34, 895 (1972).

${ }^{16}$ R. Lipowsky and G. Gompper, Phys. Rev. B 29, 5213 (1984).

${ }^{17}$ D. Sornette, Phys. Rev. B 31, 4672 (1985).

${ }^{18}$ A. A. Sobyanin and A. A. Stratonnikov, Phys. Lett. A 126, 356 (1988).

${ }^{19}$ Y. Imry, Phys. Rev. B 21, 2042 (1980).

${ }^{20}$ M. N. Barber, in Phase Transitions and Critical Phenomena, Vol. 8 edited by C. Domb and J. L. Lebowitz (Academic, New York, 1983).

${ }^{21}$ A. G. Merganov and E. N. Rumanov, Sov. Phys. Usp. 30, 293 (1987).

${ }^{22}$ R. S. Berry and D. J. Wales, Phys. Rev. Lett. 63, 1156 (1989).

${ }^{23}$ R. D. Shull, R. D. McMichael, and J. J. Ritter, Nanostructured Materials 2, 205 (1993).

${ }^{24}$ L. D. Landau, Phys. Z. Sowjetunion 12, 123 (1937); in Collected Papers of L. D. Landau, edited by D. Ter-Haar (Gordon and Breach, London, 1967), p. 236.

${ }^{25}$ J. W. Cahn and J. E. Hilliard, J. Chem. Phys. 28, 258 (1958).

${ }^{26}$ I. M. Gelfand and S. V. Fomin, Calculus of Variations (Prentice Hall, New York, 1963).

${ }^{27}$ L. D. Landau, Phys. Z. Sowjetunion 11, 26,545 (1937); in Collected Papers of L. D. Landau, edited by D. Ter-Haar (Cordon and Breach, London, 1967), pp. 193,209.

${ }^{28}$ A. Umantsev and G. B. Olson, Phys. Rev. E 48, 4229 (1993).

${ }^{29}$ A. Umantsev and G. B. Olson, Phys. Rev. A 46, R6132 (1992).

${ }^{30}$ J. P. Rose and R. S. Berry, J. Chem Phys. 96, 517 (1992).

${ }^{31}$ S. Sawada and S. Sugano, Z. Phys. D 12, 189 (1989); 14, 247 (1989).

${ }^{32}$ T. Suzuki and K. Takahashi, Mater. Trans. JIM 33, 184 (1992).

${ }^{33}$ M. Born and K. Huang, Dynamical Theory of Crystal Lattices (Clarendon, Oxford, 1954).

${ }^{34}$ L. D. Landay and E. M. Lifshitz, Theory of Elastisity (Pergamon, Oxford, 1958).

${ }^{35}$ P. Cheyssac, R. Kofman, and R. Garrigos, Phys. Scr. 38, 164 (1988).

${ }^{36}$ Y-W. Kim, H-M. Lin, and T. F. Kelly, Acta Metall. 37, 247 (1989).

${ }^{37}$ A Umantsev, in Proceedings of the International Conference PTM'94 (PA, USA, 1994). 